\title{
Olive Twig and Branch Dieback: Etiology, Incidence, and Distribution in California
}

J. R. Úrbez-Torres, Pacific Agri-Food Research Centre, Agriculture and Agri-Food Canada, 4200 Highway 97, Box 5000, Summerland, British Columbia V0H1Z0, Canada; F. Peduto, Department of Plant Pathology, University of California Davis, Davis, CA 95616, USA; P. M. Vossen, University of California Cooperative Extension Sonoma County, Santa Rosa, CA 95403, USA; W. H. Krueger, University of California Cooperative Extension Glenn County, Orland, CA 95963, USA; and W. D. Gubler, Department of Plant Pathology, University of California Davis, Davis, CA 95616, USA

\begin{abstract}
Úrbez-Torres, J. R., Peduto, F., Vossen, P. M., Krueger, W. H., and Gubler, W. D. 2013. Olive twig and branch dieback: Etiology, incidence, and distribution in California. Plant Dis. 97:231-244.

Eighteen different fungal species were isolated from symptomatic wood of olive trees (Olea europaea) affected by twig and branch dieback in California and identified by means of morphological characters and multigene sequence analyses of the internal transcribed spacer (ITS) region (ITS1-5.8S-ITS2), a partial sequence of the $\beta$-tubulin gene, and part of the translation elongation factor $1-\alpha$ gene $(E F 1-\alpha)$. These species included Diaporthe viticola, Diatrype oregonensis, Diatrype stigma, Diplodia mutila, Dothiorella iberica, Lasiodiplodia theobromae, Phaeomoniella chlamydospora, Phomopsis sp. group 1, Phomopsis sp. group 2, and Schizophyllum commune, which are for the first time reported to occur in olive trees; Eutypa lata, Neofusicoccum

lum, which are for the first time reported to occur in olive trees in the United States; and Botryosphaeria dothidea, Diplodia seriata, Neofusicoccum mediterraneum, and Trametes versicolor, which have been previously reported in olive trees in California. Pathogenicity studies conducted in olive cultivars Manzanillo and Sevillano showed $N$. mediterraneum and Diplodia mutila to be the most virulent species and Diatrype stigma and D. oregonensis the least virulent when inoculated in olive branches. Intermediate virulence was shown for the rest of the taxa. This study demystifies the cause of olive twig and branch dieback and elucidates most of the fungal pathogens responsible for this disease in California.
\end{abstract} luteum, Neofusicoccum vitifusiforme, and Phaeoacremonium aleophi-
The evergreen tree European olive (Olea europaea L.), with over 9.2 million hectares cultivated and more than 19.3 million metric tons harvested in 2009 worldwide, is one of the most extensively planted fruit crops in the world (9). Although the Mediterranean Basin accounts for $95 \%$ of the world's olive production, olive trees are also cultivated in several regions of the world with Mediterranean and/or temperate climates, including China, Australia, New Zealand, South Africa, Argentina, Uruguay, Chile, Peru, El Salvador, Mexico, and California in the United States (9).

Olives were introduced into California from northern Mexico by Franciscan padres in the early 1770 s, with the first trees being planted at the San Diego de Alcalá Mission, now city of San Diego (7). Nowadays, the California olive industry produces $99 \%$ of the olives in the United States and comprises over 21,000 ha of bearing trees. In 2010, California's olive production was over 195,000 metric tons, which represented a crop valued at over $\$ 113$ million (41). Historically, the California olive industry has been based almost entirely on the production of canned ripe olives, with about $90 \%$ of the total production being used for canning packs, including ripe (whole or pitted), green-ripe (whole or pitted), and sliced, chopped, wedged, and broken (all pitted) (7). The introduction of novel super-high-density olive varieties, on the other hand, has recently raised the interest among growers in new plantations for the production of olive oil. As a result, over 7,000 ha of olive trees have been planted in

Corresponding author: W. D. Gubler, E-mail: wdgubler@ucdavis.edu

* The $\boldsymbol{e}$-Xtra logo stands for "electronic extra" and indicates that Figures 2 and 3 appear in color online.

Accepted for publication 27 August 2012.

http://dx.doi.org/10.1094/PDIS-04-12-0390-RE

(C) 2013 The American Phytopathological Society
California since 2005, of which about $80 \%$ are super-highdensity olive tree varieties (50).

Olive trees are known to be drought resistant and hardy, suffering from few major disease problems (22). Among all diseases affecting olives, dalmatian disease caused by Botryosphaeria dothidea (Moug.:Fr.) Ces. \& De Not., olive anthracnose caused by Colletotrichum acutatum J.H. Simmonds and/or Colletotrichum gloeosporioides (Penz.) Penz. \& Sacc., olive knot caused by Pseudomonas syringae pv. savastanoi (Smith) Young, Dye \& Wilkie, peacock spot (synonyms: bird's eye spot and olive leaf spot) caused by Spilocaea oleaginea (Castagne) S. Hughes, and Verticillium wilt caused by Verticillium dahliae Kleb. are probably the most economically important and extensively studied diseases of olives worldwide $(13,18,33)$. Less studied, olive twig and branch dieback symptoms are characterized by dead twigs in the affected parts of the tree, which are generally associated with the decline of entire young stems and/or older branches $(25,27)$. Cross-sections of affected tree parts (stems, branches, and/or trunk) reveal the presence of perennial cankers in woody tissues, which diminishes water and nutrient movement through both xylem and phloem. Characteristic dieback symptoms appear when water and nutrient demand exceeds the conductive capacity of the vascular tissues. Eventually, death of scaffold branches or the entire tree occurs when the growth of the cankers cuts off vascular flow (2). However, only a few studies on the etiology of olive twig and branch dieback can be found throughout the literature. Malathrakis (16) reported olive cankers and consequent dieback associated with the fungus Phoma incompta Sacc. \& Martelli in Greece in 1979. Between the late 1980s and early 1990s, Cytospora oleina Berl. and Eutypa lata (Pers.) Tul. \& C. Tul. were identified as the causal agents of olive branch dieback in Greece $(26,27)$. During the 2000s, several fungi were associated with branch dieback, stem canker, and/or shoot necrosis of olive trees, including E. lata (35), a Phoma sp. (24), and several Botryosphaeriaceae species $(14,20,25,32)$. Most recently, B. dothidea, Diplodia seriata De Not., and Neofusicoccum mediterraneum Crous, M.J. Wingf. \& 
A.J.L. Phillips were reported to cause branch dieback and necrosis, blight, and eventual death of olive shoots in California (20).

In California, olive twig dieback has long been known to commonly occur in orchards throughout the San Joaquin Valley $(30,33)$. The disease has been historically associated with a Diplodia sp., which gave Diplodia dieback its name among California growers (33). However, both fungus and symptoms were found most of the time associated with either sunburned areas or declining olive tree parts affected by Verticillium wilt and/or olive knot $(20,30,33)$, and thus, very little importance was given to olive twig dieback in the state. Nevertheless, in the past few years, branch canker and twig dieback of olive trees has become a major concern among growers from the main olive-producing areas in California, and diseased samples of both young and mature trees have been continuously submitted to our laboratory.

Perennial cankers and consequent dieback are known to cause a direct impact on the health of many economically important woody perennial crops worldwide, reducing both yield and tree longevity $(2,8)$. In contrast, the lack of information currently available on the etiology of olive twig and branch dieback in California makes it difficult at the present time to assess the significance of this disease in the state. Therefore, the objectives of this study were to (i) determine the incidence and geographic distribution of olive twig and branch dieback in California by conducting field surveys, (ii) identify the different fungal species associated with the disease by means of morphological and molecular studies, and (iii) evaluate the pathogenicity of the different fungi in the two most common olive cultivars, Manzanillo and Sevillano, planted in the state.

\section{Materials and Methods}

Field surveys and fungal isolations. From October 2008 to September 2009, field surveys were conducted throughout the main olive-production areas in California, including Butte, Fresno, Glenn, Madera, Merced, Napa, Riverside, Sacramento, Santa Bar-

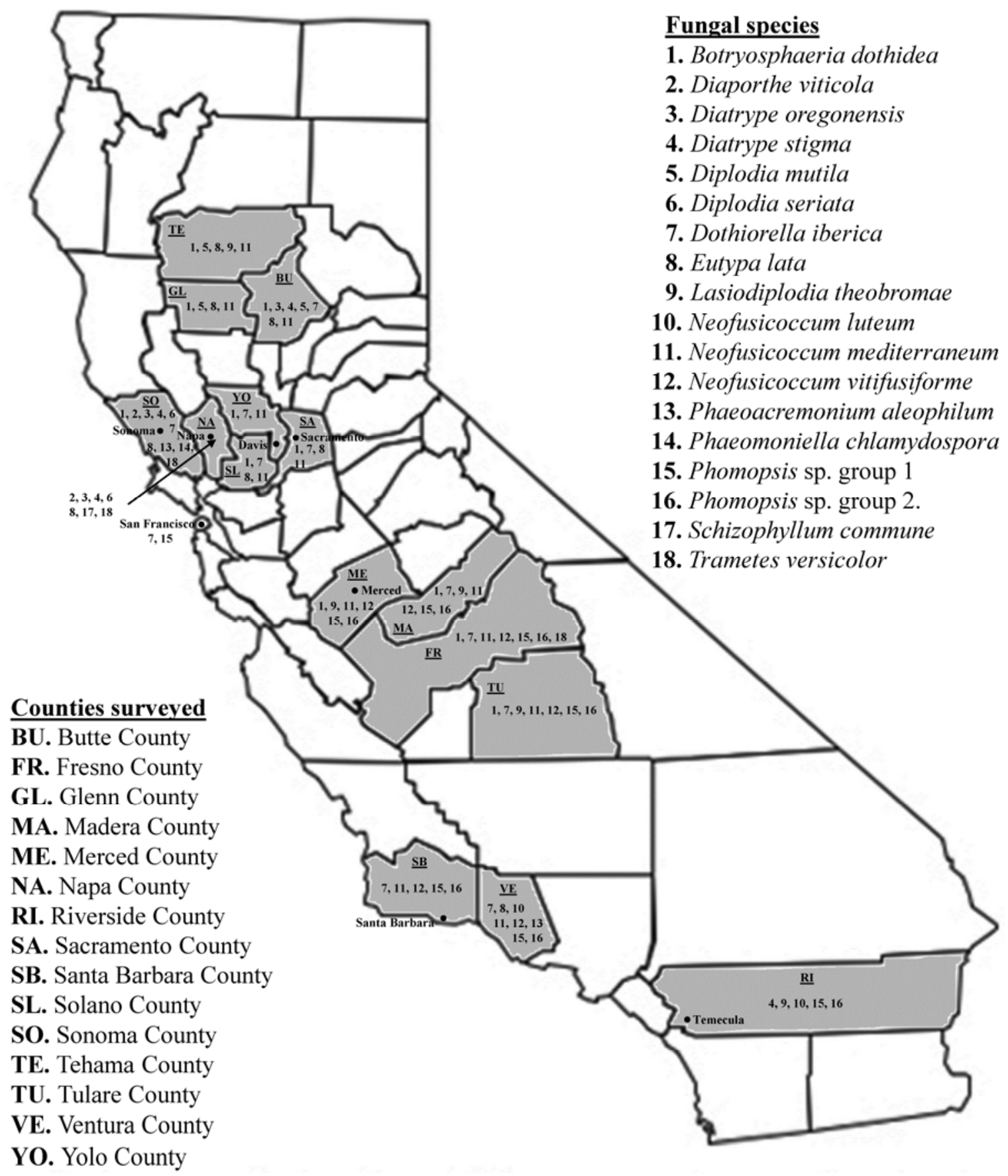

Fig. 1. Geographical distribution of fungal species on olive trees in California associated with olive twig and branch disease. 
bara, Solano, Sonoma, Tehama, Tulare, Ventura, and Yolo counties (Fig. 1). In total, 803 samples were collected from 59 mature orchards (15 years old and older). Samples included trunks, branches, and twigs collected from trees showing characteristic dieback symptoms. Samples were collected from the most prevalent olive cultivars grown in California, including Ascolano, Manzanillo, Mission, and Sevillano. Additionally, 61 samples were collected from olive trees located in urban landscape sites in the cities of Davis, Merced, Napa, Sacramento, San Francisco, San Luis Obispo, Santa Barbara, Sonoma, and Temecula (Fig. 1). Diseased samples were first inspected for the presence of fungal fruiting structures (e.g., pycnidia, perithecia, pseudothecia, etc.) in the laboratory using a Leica MZ95 (Leica Microsystems GmbH, Wetzlar, Germany) stereo microscope. Thereafter, the outer bark of the samples was peeled off, and samples (including both sapwood and heartwood) were surface sterilized by submerging them in $0.5 \%$ sodium hypochlorite for $5 \mathrm{~min}$. After air drying, sapwood tissue was shaved away to expose the margin between the cankered and healthy tissue; then the exposed wood was sprayed with $95 \%$ etha- nol and briefly flamed. Small pieces of tissue (approximately 25 $\mathrm{mm}^{2}$ ) were placed on $85-\mathrm{mm}$-diameter petri dishes containing $4 \%$ potato dextrose agar (PDA) (Difco, Detroit, MI) amended with tetracycline hydrochloride $(0.01 \%)$ (Sigma-Aldrich, St. Louis, MO) (PDA-tet). Cultures were incubated at room temperature (24 $\pm 2^{\circ} \mathrm{C}$ ) until fungal colonies were observed. The most prevalent fungal taxa observed from the symptomatic wood tissue were then individually transferred to fresh PDA-tet. Pure cultures of the different fungi were obtained by hyphal tip from colony margins and placed on fresh PDA. Pure fungal colonies were then incubated at ambient laboratory light and temperature conditions.

Morphological characterization. Fungal species were first identified tentatively to genus based on colony characteristics (color, mycelium growth speed, and type and shape of the colony) after 3 or 4 week's incubation. When available, both pycnidial and conidial characteristics (shape, size, color, presence or absence of septum) from colonies forming in vitro were recorded. For those cultures lacking sporulation, pycnidia production was induced by placing 5-mm-diameter mycelium plugs over double-autoclaved

Table 1. Isolates from olive trees from California included in the phylogenetic analyses

\begin{tabular}{|c|c|c|c|c|c|c|}
\hline \multirow[b]{2}{*}{ Species } & \multirow[b]{2}{*}{ Isolate } & \multirow[b]{2}{*}{ Cultivar } & \multirow[b]{2}{*}{ Origin } & \multicolumn{3}{|c|}{ GenBank accession no. } \\
\hline & & & & ITS $^{\mathbf{y}}$ & EF1- $\alpha^{\mathrm{z}}$ & $\beta$-tubulin \\
\hline Botryosphaeria dothidea & UCD30-Oe & Sevillano & Butte Co. & JX515699 & JX515747 & JX515667 \\
\hline Botryosphaeria dothidea & UCD62-Oe & Mission & Butte Co. & JX515700 & JX515748 & JX515668 \\
\hline Diaporthe viticola & UCD316-Oe & Sevillano & Napa Co. & JX515701 & JX515749 & - \\
\hline Diaporthe viticola & UCD327-Oe & Manzanillo & Napa Co. & JX515702 & JX515750 & - \\
\hline Diatrype oregonensis & UCD60-Oe & Mission & Butte Co. & JX515703 & JX515751 & JX515669 \\
\hline Diatrype stigma & UCD23-Oe & Mission & Butte Co. & JX515704 & JX515752 & JX515670 \\
\hline Diatrype stigma & UCD24-Oe & Mission & Butte Co. & JX515705 & JX515753 & JX515671 \\
\hline Diatrype stigma & UCD356-Oe & Sevillano & Riverside Co. & JX515706 & - & JX515672 \\
\hline Diatrype stigma & UCD357-Oe & Sevillano & Riverside Co. & JX515707 & - & JX515673 \\
\hline Diplodia mutila & UCD127-Oe & Manzanillo & Glenn Co. & JX515745 & JX515786 & JX515674 \\
\hline Diplodia mutila & UCD147-Oe & Sevillano & Glenn Co. & JX515746 & JX515787 & JX515675 \\
\hline Diplodia seriata & UCD301-Oe & Manzanillo & Napa Co. & JX515708 & JX515754 & JX515676 \\
\hline Diplodia seriata & UCD340-Oe & Mission & Napa Co. & JX515709 & JX515755 & JX515677 \\
\hline Dothiorella iberica & UCD50-Oe & Mission & Butte Co. & JX515710 & JX515756 & JX515678 \\
\hline Dothiorella iberica & UCD673-Oe & Mission & Yolo Co. & JX515711 & JX515757 & JX515679 \\
\hline Eutypa lata & UCD143-Oe & Manzanillo & Glenn Co. & JX515712 & JX515758 & JX515680 \\
\hline Eutypa lata & UCD144-Oe & Manzanillo & Glenn Co. & JX515713 & JX515759 & JX515681 \\
\hline Eutypa lata & UCD318-Oe & Sevillano & Napa Co. & JX515714 & JX515760 & JX515682 \\
\hline Eutypa lata & UCD319-Oe & Sevillano & Napa Co. & JX515715 & JX515761 & JX515683 \\
\hline Lasiodiplodia theobromae & UCD375-Oe & Mission & Riverside Co. & JX515716 & JX515762 & JX515684 \\
\hline Lasiodiplodia theobromae & UCD527-Oe & Sevillano & Tehama Co. & JX515717 & JX515763 & JX515685 \\
\hline Neofusicoccum luteum & UCD360-Oe & Mission & Riverside Co. & JX515718 & JX515764 & JX515686 \\
\hline Neofusicoccum luteum & UCD369-Oe & Manzanillo & Riverside Co. & JX515719 & JX515765 & JX515687 \\
\hline Neofusicocum mediterraneum & UCD1-Oe & Sevillano & Butte Co. & JX515720 & JX515766 & JX515688 \\
\hline Neofusicocum mediterraneum & UCD22-Oe & Mission & Butte Co. & JX515721 & JX515767 & JX515689 \\
\hline Neofusicocum mediterraneum & UCD453-Oe & Manzanillo & Sacramento Co. & JX515722 & JX515768 & JX515690 \\
\hline Neofusicocum mediterraneum & UCD679-Oe & Mission & Yolo Co. & JX515723 & JX515769 & JX515691 \\
\hline Neofusicoccum vitifusiforme & UCD622-Oe & Manzanillo & Ventura Co. & JX515724 & JX515770 & JX515692 \\
\hline Neofusicoccum vitifusiforme & UCD624-Oe & Manzanillo & Ventura Co. & JX515725 & JX515771 & JX515693 \\
\hline Neofusicoccum vitifusiforme & UCD630-Oe & Sevillano & Ventura Co. & JX515726 & JX515772 & JX515694 \\
\hline Phaeoacremonium aleophilum & UCD228-Oe & Manzanillo & Napa Co. & JX515727 & JX515773 & JX515695 \\
\hline Phaeoacremonium aleophilum & UCD468-Oe & Mission & Sonoma Co. & JX515728 & JX515774 & JX515696 \\
\hline Phaeomoniella chlamydospora & UCD306-Oe & Manzanillo & Napa Co. & JX515729 & JX515775 & JX515697 \\
\hline Phaeomoniella chlamydospora & UCD471-Oe & Mission & Sonoma Co. & JX515730 & JX515776 & JX515698 \\
\hline Phomopsis sp. & UCD181-Oe & Manzanillo & Madera Co. & JX515731 & JX515777 & - \\
\hline Phomopsis sp. & UCD182-Oe & Manzanillo & Madera Co. & JX515732 & JX515778 & - \\
\hline Phomopsis sp. & UCD213-Oe & Sevillano & Madera Co. & JX515733 & JX515779 & - \\
\hline Phomopsis sp. & UCD233-Oe & Manzanillo & Merced Co. & JX515734 & JX515780 & - \\
\hline Phomopsis sp. & UCD237-Oe & Manzanillo & Merced Co. & JX515735 & JX515781 & - \\
\hline Phomopsis sp. & UCD248-Oe & Sevillano & Merced Co. & JX515736 & JX515782 & - \\
\hline Phomopsis sp. & $\mathrm{UCD} 278-\mathrm{Oe}$ & Mission & Merced Co. & JX515737 & JX515783 & - \\
\hline Phomopsis sp. & UCD580-Oe & Manzanillo & Tulare Co. & JX515738 & JX515784 & - \\
\hline Schizophyllum commune & UCD296-Oe & Sevillano & Napa Co. & JX515739 & - & - \\
\hline Schizophyllum commune & UCD304-Oe & Manzanillo & Napa Co. & JX515740 & - & - \\
\hline Schizophyllum commune & UCD309-Oe & Manzanillo & Napa Co. & JX515741 & - & - \\
\hline Trametes versicolor & UCD311-Oe & Manzanillo & Napa Co. & JX515742 & - & - \\
\hline Trametes versicolor & UCD312-Oe & Manzanillo & Napa Co. & JX515743 & - & - \\
\hline Trametes versicolor & UCD313-Oe & Mission & Napa Co. & JX515744 & - & - \\
\hline
\end{tabular}

${ }^{y}$ rDNA Internal Transcribed Spacer region.

${ }^{\text {z }}$ Translation Elongation Factor. 
pine needles placed on $2 \%$ water agar (Difco) as described by Úrbez-Torres et al. (46). Pycnidia were mounted in water, and conidial masses were observed by bright field microscopy using a Leica DMLB (Leica Microsystems $\mathrm{GmbH}$ ) compound microscope. Images were recorded with a Leica DFC480 digital camera.

DNA extraction, amplification, and phylogenetic analyses. Representative isolates of each fungal taxa identified using morphological characteristics were selected for molecular identification (Table 1). Fungal isolates were cultured on PDA and grown for 2 weeks. Total genomic DNA was extracted using the DNeasy Plant Mini Kit (Qiagen Inc., Valencia, CA). Oligonucleotide primers ITS1 and ITS4, Bt2a and Bt2b, and EF1-728F and EF1-986R were used to amplify the internal transcribed spacer (ITS) ITS15.8S-ITS2 ribosomal DNA region, a portion of the beta-tubulin (BT) gene, and part of the translation elongation factor (EF1- $\alpha)$ gene, respectively $(5,10,51)$. ITS, BT, and EF1- $\alpha$ amplification reactions were carried out using a thermal cycler (PTC-100, MJ Research, Watertown, MA) $(5,10,51)$. Amplification products were purified using QIAquick PCR purification Kit (Qiagen Inc.). Both strands of the ITS, BT, and EF1- $\alpha$ amplicons were sequenced using an ABI Prism 377 DNA Sequencer (Perkin-Elmer, Norwalk, CN) at the Division of Biological Sciences sequencing facility at University of California, Davis. Fungal sequences were edited and assembled using Sequencher version 4.1 (Gene Codes, Ann Arbor, $\mathrm{MI}$ ) and were aligned using the ClustalW multiple alignment program (34). Manual adjustments of sequence alignment were carried out using BioEdit Sequence Alignment Editor Version 7.0.8. (Ibis Biosciences, Carslbad, CA). Fungal sequences, including those from ex-type specimens of taxa, when possible, were selected from GenBank (Table 2) based on their high similarity to query sequences using MegaBLAST.

Phylogenetic analyses were performed on individual datasets on the fungal families Botryosphaeriaceae (ITS, BT, and EF1- $\alpha$ ) and Diatrypaceae (ITS and BT), and on the genera DiaporthePhomopsis (ITS and EF1- $\alpha$ ), Phaeoacremonium (ITS and BT), Phaeomoniella (ITS and BT), and Schizophyllum and Trametes (ITS). All tree topologies were visually compared for congruence in order to permit concatenation of data sets. Multigene phylogenetic analyses were then performed on the Botryosphaeriaceae (ITS+BT+EF1- $\alpha$ ), Diatrypaceae (ITS+BT), Diaporthe-Phomopsis (ITS+EF1- $\alpha$ ), Phaeoacremonium (ITS+BT), and Phaeomoniella (ITS+BT) (single family and/or genera trees based on both individual and multigene datasets not shown). Phylogenetic analysis of the ITS region, including all taxa, and using the Basidiomycetes genera Schizophyllum and Trametes as an out-group, was performed to illustrate all fungal species identified from olive trees

Table 2. Isolates from GenBank included in the phylogenetic analyses

\begin{tabular}{|c|c|c|c|c|c|c|c|}
\hline \multirow[b]{2}{*}{ Species } & \multirow[b]{2}{*}{ Isolate $^{w}$} & \multirow[b]{2}{*}{ Host } & \multirow[b]{2}{*}{ Origin } & \multirow[b]{2}{*}{ Collector } & \multicolumn{3}{|c|}{ GenBank accession no. } \\
\hline & & & & & ITS $^{x}$ & EF1- $\alpha^{y}$ & $\beta$-tubulin \\
\hline Botryosphaeria dothidea & CMW8000 & Prunus sp. & Switzerland & B. Slippers & AY236949 & AY236898 & AY236927 \\
\hline Botryosphaeria dothidea & UCD1064So & Vitis vinifera & California, USA & J.R. Úrbez-Torres & DQ233600 & GU294733 & DQ233621 \\
\hline $\begin{array}{l}\text { Cryptosphaeria } \\
\text { pullmanensis }\end{array}$ & UCD2371NV & Vitis vinifera & Nevada, USA & J.R. Úrbez-Torres & GQ293966 & $\mathrm{n} / \mathrm{a}^{\mathrm{z}}$ & GQ294011 \\
\hline Cryptovalsa ampelina & UCD311Ma & Vitis vinifera & California, USA & J.R. Úrbez-Torres & GQ293906 & $\mathrm{n} / \mathrm{a}$ & GQ293975 \\
\hline Diaporthe ambigua & CBS 114015 & Pyrus communis & South Africa & S. Denman & AF230767 & GQ250299 & $\mathrm{n} / \mathrm{a}$ \\
\hline Diaporthe ambigua & CBS 123210 & $\begin{array}{l}\text { Foeniculum } \\
\text { vulgare }\end{array}$ & Portugal & J.M. Santos & EU814479 & GQ250300 & $\mathrm{n} / \mathrm{a}$ \\
\hline Diaporthe ambigua & CBS 123211 & $\begin{array}{l}\text { Foeniculum } \\
\text { vulgare }\end{array}$ & Portugal & J.M. Santos & EU814478 & GQ250301 & $\mathrm{n} / \mathrm{a}$ \\
\hline Diaporthe angelicae & CBS 111592 & $\begin{array}{l}\text { Heracleum } \\
\text { sphondylium }\end{array}$ & Austria & W. Jacklitsch & AY196779 & GQ250302 & $\mathrm{n} / \mathrm{a}$ \\
\hline Diaporthe aspalathi & CBS 117169 & Aspalathus linearis & South Africa & J.C.J. van Rensburg & DQ286275 & DQ286249 & $\mathrm{n} / \mathrm{a}$ \\
\hline Diaporthe crotalariae & CBS 162.33 & $\begin{array}{l}\text { Crotalaria } \\
\text { spectabilis }\end{array}$ & North America & G.F. Weber & FJ889445 & GQ250307 & $\mathrm{n} / \mathrm{a}$ \\
\hline Diaporthe helianthi & CBS 592.81 & Helianthus anпииs & Serbia & $\begin{array}{l}\text { M. Muntanola- } \\
\text { Cvetkovic }\end{array}$ & AY705842 & GQ250308 & $\mathrm{n} / \mathrm{a}$ \\
\hline Diaporthe melonis & CBS 507.78 & Cucumis melo & Texas, USA & $\begin{array}{l}\text { I. Beraha \& M.J. } \\
\text { O'Brein }\end{array}$ & FJ889447 & GQ250314 & $\mathrm{n} / \mathrm{a}$ \\
\hline Diaporthe neotheicola & CBS 123208 & $\begin{array}{l}\text { Foeniculum } \\
\text { vulgare }\end{array}$ & Portugal & A.J.L. Phillips & EU814480 & GQ250315 & $\mathrm{n} / \mathrm{a}$ \\
\hline Diaporthe vaccinii & CBS 160.32 & $\begin{array}{l}\text { Oxycoccus } \\
\text { macrocarpus }\end{array}$ & Oregon, USA & H.F. Bain & AY952141 & GQ250326 & $\mathrm{n} / \mathrm{a}$ \\
\hline Diaporthe viticola & CBS 113201 & Vitis vinifera & Portugal & A.J.L. Phillips & AY485750 & GQ250327 & $\mathrm{n} / \mathrm{a}$ \\
\hline Diaporthe viticola & 111 & Vitis vinifera & Portugal & E. Diogo & GQ250200 & GQ250329 & $\mathrm{n} / \mathrm{a}$ \\
\hline Diaporthe viticola & $\mathrm{Di}-\mathrm{C} 006 / 2$ & $\begin{array}{l}\text { Hydrangea } \\
\text { macrophylla }\end{array}$ & Portugal & A. Alves & GQ250199 & GQ250328 & $\mathrm{n} / \mathrm{a}$ \\
\hline Diatrype oregonensis & DRO102 & Prunus armeniaca & California, USA & F.P. Trouillas & GQ293933 & $\mathrm{n} / \mathrm{a}$ & GQ293998 \\
\hline Diatrype oregonensis & CA117 & Vitis vinifera & California, USA & F.P. Trouillas & GQ293934 & $\mathrm{n} / \mathrm{a}$ & GQ293996 \\
\hline Diatrype sp. & CDB016 & Vitis vinifera & California, USA & F.P. Trouillas & GQ293950 & $\mathrm{n} / \mathrm{a}$ & GQ294001 \\
\hline Diatrype stigma & CA074 & Vitis vinifera & California, USA & F.P. Trouillas & GQ293944 & $\mathrm{n} / \mathrm{a}$ & GQ294005 \\
\hline Diatrype stigma & DCHR5 & Vitis vinifera & California, USA & F.P. Trouillas & GQ293945 & $\mathrm{n} / \mathrm{a}$ & GQ294006 \\
\hline Diatrype stigma & DCASH200 & Quercus sp. & California, USA & F.P. Trouillas & GQ293947 & $\mathrm{n} / \mathrm{a}$ & GQ294003 \\
\hline Diatrype whitemanensis & DDES500 & Acer macrophyllum & California, USA & F.P. Trouillas & GQ293952 & $\mathrm{n} / \mathrm{a}$ & GQ294009 \\
\hline $\begin{array}{l}\text { Diatrypella } \\
\text { verrucaeformis }\end{array}$ & DCH500 & Quercus sp. & California, USA & F.P. Trouillas & GQ293926 & $\mathrm{n} / \mathrm{a}$ & GQ293991 \\
\hline Diplodia mutila & CBS 112553 & Vitis vinifera & Portugal & A.J.L. Phillips & AY259093 & AY573219 & DQ458850 \\
\hline Diplodia mutila & UCD288Ma & Vitis vinifera & California, USA & J.R. Úrbez-Torres & DQ008313 & EU012411 & DQ008336 \\
\hline Diplodia seriata & CBS 112555 & Vitis vinifera & Portugal & A.J.L. Phillips & AY259094 & AY573220 & DQ458856 \\
\hline Diplodia seriata & UCD244Ma & Vitis vinifera & California, USA & J.R. Úrbez-Torres & DQ008314 & EU012406 & DQ008337 \\
\hline Dothiorella iberica & CBS 115041 & Quercus ilex & Spain & J. Luque & AY573202 & AY573222 & EU673096 \\
\hline & & & & & & (continu & n next page) \\
\hline
\end{tabular}

\footnotetext{
${ }^{\mathrm{w}}$ Isolate numbers in bold represent ex-type specimens.

${ }^{x}$ rDNA Internal Transcribed Spacer region.

y Translation Elongation Factor.

${ }^{\mathrm{z}} \mathrm{n} / \mathrm{a}=$ not available
} 
in California. Single and multigene phylogenetic analyses were performed with PAUP version 4.0b10 (31) using maximum parsimony (MP) with a heuristic search and 1,000 random addition sequence replicates. Trees were rooted using the midpoint rooting option, and tree bisection-reconnection (TBR) was used as the branch swapping algorithm. Branches of zero length were collapsed, and all multiple, equally parsimonious trees were saved. Alignment gaps were treated as missing data, and all characters were unordered and equally weighted. Measures including tree length, consistency index (CI), and retention index (RI) were calculated. Bootstrap support (BS) was estimated using 1,000 replicates to assess the robustness of each clade. Fungal sequences from olive from California were deposited into GenBank, and representative isolates are maintained in the collection of the Plant Pathology Department at the University of California, Davis (Table 1).

Pathogenicity tests. In order to determine which fungal species isolated from cankers of olive trees were important pathogens and to differentiate them from potential saprophytes, secondary colonizers, and/or weak parasites, one representative isolate of each fungal species identified from symptomatic wood tissue was selected to determine pathogenicity. Pathogenicity tests were conducted on mature Manzanillo and Sevillano olive trees located in an experimental commercial orchard at the University of California
Nickels Soil Laboratory Field Station in Arbuckle, CA in October of 2009. Olive branches (2- and 3-year-old) from both cultivars were individually inoculated with fungal species. The distal end of each tree branch was inoculated by placing a 5-mm-diameter mycelium plug from a 7-day-old PDA culture in a wound made with a 5-mm-diameter cork-borer. Wounds were sealed with petroleum jelly and protected with Parafilm. Fungal treatments were compared to control treatments inoculated with noncolonized media plugs, also sealed with petroleum jelly and protected by Parafilm. Ten branches per fungal isolate ( 1 branch per tree) and per olive cultivar were used, and inoculations were arranged in a completely randomized design. Samples were collected after 6 months of incubation and returned to the laboratory for assessment of canker length. Samples were surface disinfected as previously described. After air drying, samples were split longitudinally through the point of inoculation, and the extent of both acropetal and basipetal vascular discoloration from the point of inoculation was measured. In an attempt to fulfill Koch's postulates, small pieces of necrotic tissue from the edge of each lesion were cut and placed on PDA-tet to recover the inoculated fungus.

Data from the pathogenicity test were analyzed using SAS (Version 9.1.3; SAS Institute, Cary, NC). Homogeneity of variance was tested using Levene's test. Residuals were visually inspected for

Table 2. (continued from previous page)

\begin{tabular}{|c|c|c|c|c|c|c|c|}
\hline \multirow[b]{2}{*}{ Species } & \multirow[b]{2}{*}{ Isolate $^{w}$} & \multirow[b]{2}{*}{ Host } & \multirow[b]{2}{*}{ Origin } & \multirow[b]{2}{*}{ Collector } & \multicolumn{3}{|c|}{ GenBank accession no. } \\
\hline & & & & & ITS $^{x}$ & EF1- $\alpha^{y}$ & $\beta$-tubulin \\
\hline $\begin{array}{l}\text { Dothiorella } \\
\text { sarmentorum }\end{array}$ & IMI63581b & Ulmus sp. & United Kingdom & E.A. Ellis & AY573212 & AY573235 & EU673102 \\
\hline Eutypa lata & UCDE7 & Vitis vinifera & California, USA & P.E. Rolshausen & DQ006944 & $\mathrm{n} / \mathrm{a}$ & DQ00700 \\
\hline Eutypa lata & UCDE31 & Vitis vinifera & California, USA & P.E. Rolshausen & DQ006933 & $\mathrm{n} / \mathrm{a}$ & DQ006990 \\
\hline Eutypa lata & UCDE38 & Vitis vinifera & California, USA & P.E. Rolshausen & DQ006935 & $\mathrm{n} / \mathrm{a}$ & DQ006992 \\
\hline Eutypa lata & UCD2275MO & Vitis vinifera & Missouri, USA & J.R. Úrbez-Torres & HQ288221 & $\mathrm{n} / \mathrm{a}$ & HQ288300 \\
\hline Eutypa leptoplaca & D-Rsn-200 & Vitis vinifera & California, USA & F.P. Trouillas & AY 684237 & $\mathrm{n} / \mathrm{a}$ & AY 684212 \\
\hline $\begin{array}{l}\text { Lasiodiplodia } \\
\text { theobromae }\end{array}$ & CBS 164.96 & $\begin{array}{l}\text { Fruit along the } \\
\text { coral reef coast }\end{array}$ & $\begin{array}{l}\text { Papua New } \\
\text { Guinea }\end{array}$ & $\mathrm{n} / \mathrm{a}$ & AY640255 & AY640258 & EU673110 \\
\hline $\begin{array}{l}\text { Lasiodiplodia } \\
\text { theobromae }\end{array}$ & CAA006 & Vitis vinifera & California, USA & T.J. Michailides & DQ458891 & DQ458876 & DQ458859 \\
\hline $\begin{array}{l}\text { Lasiodiplodia } \\
\text { theobromae }\end{array}$ & UCD205Co & Vitis vinifera & California, USA & J.R. Úrbez-Torres & DQ008310 & EU012398 & DQ008333 \\
\hline Neofusicoccum luteum & CBS 110299 & Vitis vinifera & Portugal & A.J.L. Phillips & AY259091 & AY573217 & DQ458848 \\
\hline $\begin{array}{l}\text { Neofusicoccum } \\
\text { mediterraneum }\end{array}$ & CBS 121718 & Eucalyptus sp. & Greece & $\begin{array}{l}\text { Crous, Wingfield \& } \\
\text { Phillips }\end{array}$ & GU251176 & GU251308 & GU251836 \\
\hline $\begin{array}{l}\text { Neofusicoccum } \\
\text { mediterraneum }\end{array}$ & UCD720SJ & Vitis vinifera & California, USA & J.R. Úrbez-Torres & GU799452 & GU799483 & GU799475 \\
\hline Neofusicoccum parvum & CMW9081 & Populus nigra & New Zealand & G.J. Samuels & AY236943 & AY236888 & AY236917 \\
\hline Neofusicoccum ribis & CMW7772 & Ribes sp. & New York, USA & $\begin{array}{l}\text { B. Slippers \& G. } \\
\text { Hudler }\end{array}$ & AY236935 & AY236877 & AY236906 \\
\hline $\begin{array}{l}\text { Neofusicoccum } \\
\text { vitifusiforme }\end{array}$ & STE-U 5252 & Vitis vinifera & South Africa & J.M. van Niekerk & AY343383 & AY343343 & $\mathrm{n} / \mathrm{a}$ \\
\hline $\begin{array}{l}\text { Neofusicoccum } \\
\text { vitifusiforme }\end{array}$ & UCD2183MO & Vitis vinifera & Missouri, USA & $\begin{array}{r}\text { K. Striegler \& } \\
\text { G.M. Leavitt }\end{array}$ & HQ288214 & HQ288258 & HQ288293 \\
\hline $\begin{array}{l}\text { Phaeoacremonium } \\
\text { aleophilum }\end{array}$ & CBS 246.91 & Vitis vinifera & Serbia & $\begin{array}{l}\text { M. Muntanola- } \\
\text { Cvetkovic }\end{array}$ & AF017651 & $\mathrm{n} / \mathrm{a}$ & AF286811 \\
\hline $\begin{array}{l}\text { Phaeoacremonium } \\
\text { aleophilum }\end{array}$ & CBS 100397 & Vitis vinifera & Italy & $\mathrm{n} / \mathrm{a}$ & AF197981 & $\mathrm{n} / \mathrm{a}$ & AF246806 \\
\hline $\begin{array}{l}\text { Phaeoacremonium } \\
\text { angustius }\end{array}$ & CBS 249.95 & Vitis vinifera & USA & P. Larignon & AF197974 & $\mathrm{n} / \mathrm{a}$ & AF246814 \\
\hline $\begin{array}{l}\text { Phaeomoniella } \\
\text { chlamydospora }\end{array}$ & CBS 229.95 & Vitis vinifera & Italy & L. Mugnai & AF197973 & $\mathrm{n} / \mathrm{a}$ & AF253968 \\
\hline $\begin{array}{l}\text { Phaeomoniella } \\
\text { chlamydospora }\end{array}$ & STE-U 3066 & Vitis vinifera & South Africa & $\mathrm{n} / \mathrm{a}$ & AF197986 & $\mathrm{n} / \mathrm{a}$ & AF253969 \\
\hline $\begin{array}{l}\text { Phaeomoniella } \\
\text { chlamydospora }\end{array}$ & UCD2548MO & Vitis vinifera & Missouri, USA & $\begin{array}{r}\text { K. Striegler \& } \\
\text { G.M. Leavitt }\end{array}$ & HQ288241 & HQ288276 & HQ288313 \\
\hline Phomopsis dauci & CBS 315.49 & Daucus carota & Netherlands & J.A. von Arx & FJ889451 & GQ250348 & $\mathrm{n} / \mathrm{a}$ \\
\hline Phomopsis phoenicicola & CBS 161.64 & Areca catechu & India & H.C. Srivastava & FJ889452 & GQ250349 & $\mathrm{n} / \mathrm{a}$ \\
\hline Phomopsis sp. & UCD1685S1 & Vitis vinifera & California, USA & J.R. Úrbez-Torres & FJ794470 & JX515785 & $\mathrm{n} / \mathrm{a}$ \\
\hline Phomopsis sp. & CAL-5 & Vitis vinifera & California, USA & $\mathrm{n} / \mathrm{a}$ & AY745085 & AY745055 & $\mathrm{n} / \mathrm{a}$ \\
\hline Phomopsis sp. & 151 & Vitis vinifera & Portugal & E. Diogo & GQ250226 & GQ250364 & $\mathrm{n} / \mathrm{a}$ \\
\hline Phomopsis sp. & $\mathrm{Ph}-\mathrm{AC} 002$ & Acanthus sp. & Portugal & E. Diogo & GQ250216 & GQ250354 & $\mathrm{n} / \mathrm{a}$ \\
\hline Phomopsis viticola & CBS 114016 & Vitis vinifera & France & P. Larignon & AF230751 & GQ250351 & $\mathrm{n} / \mathrm{a}$ \\
\hline $\begin{array}{l}\text { Spencermartinsia } \\
\text { viticola }\end{array}$ & CBS 117009 & Vitis vinifera & Spain & J. Luque & AY905554 & AY905559 & EU673104 \\
\hline
\end{tabular}


each treatment, and when necessary a $\log 10$ transformation was used to improve homogeneity of variance. Difference in length of discoloration caused by each fungal isolate was determined by one-way analyses of variance. Treatment means were compared using Fisher's least significant difference (LSD) test at the 5\% significance level. A two-way ANOVA was performed to determine significant differences between Manzanillo and Sevillano olive cultivars.

\section{Results}

Field survey and symptoms of the disease. Olive twig and branch dieback symptoms were observed in all 15 counties surveyed and from all of the 59 orchards sampled (Table 3). Branch dieback of olive trees was observed in mature trees throughout California no matter the cultivar surveyed (Ascolano, Manzanillo, Mission, and Sevillano). Additionally, the disease was also observed in landscape olive trees located in the urban areas surveyed.

Symptoms associated with olive twig and branch dieback were characterized by scarce or abundant twig death localized in single or multiple branches, respectively (Fig. 2A to C). Death of twigs was usually associated with cankered stems and/or main branches (Fig. 2D). These cankers often extended through the branches until reaching the trunk causing dieback and eventual death of the branch and/or tree (Fig. 2E). Wedge-shaped cankers were the most common vascular symptom observed when affected branches were cross-sectioned (Fig. 2B and F). However, other vascular symptoms such as dark streaking of the wood and/or light brown tissue were observed in contrast to the healthy yellowish-green tissue (Fig. 2G and H). Cankers were also observed in young trees affecting the crown of the tree (Fig. 2I). Although cankers were found to develop mostly from pruning wounds made in the main branches (Fig. 2J and K), they were also observed to develop from both sunburned areas (Fig. 2L) and olive knot galls (Fig. 2M).

Morphological characterization and fungal incidence. Results from canker isolations along with morphological studies showed species in the families Botryosphaeriaceae Theiss. \& P.
Syd. and Diatrypaceae Nitschke and the genus Phomopsis Sacc. to be the most predominant fungi associated with branch cankers of olive trees in California.

Morphologically, the Botryosphaeriaceae fungal group was characterized by a light-green to dark-olivaceous fast-growing mycelium on PDA-tet (Fig. 3A and B). With age, most of these cultures developed black, globose fruiting bodies (pycnidia), which produced either pigmented or hyaline spores (conidia). Based on comparison with earlier literature (46) and previously identified isolates from grapevines in California, Botryosphaeriaceae fungal cultures with pigmented conidia were tentatively identified as different species including Lasiodiplodia theobromae, Diplodia seriata, Diplodia mutila, and Dothiorella species. Fungal cultures with hyaline conidia were tentatively identified as Botryosphaeria dothidea or at least three different Neofusicoccum species. Botryosphaeriaceae species were the most prevalent fungi isolated from both cankers ( $43 \%$ of the total) and twig dieback (24.2\% of the total) and were found in all counties surveyed (Table 3 ).

The Diaporthe-Phomopsis fungal group was characterized by having white to light-gray, slow-growing mycelium (Fig. 3C and D). Fungal colonies were slightly raised and some developed prominent growth rings with margins becoming black with age. Colonies produced dark, eustromatic pycnidia over time. Mucilaginous light-cream-colored cirrhi were observed from pycnidia (Fig. 3D). Cirrhi contained filiform and mostly curved conidia. All these morphological characteristics were consistent with the description of Diaporthe-Phomopsis spp. $(40,48)$; however, identification to species level was not possible based only on colony and conidia morphology. Diaporthe-Phomopsis spp. were the second most prevalent fungi isolated from both cankers $(12.9 \%$ of the total) and twig dieback ( $9.4 \%$ of the total) and were most commonly found in olive orchards located throughout the San Joaquin Valley, Central Coast, and southern California (Table 3).

Diatrypaceae species were characterized by having white to white-cream flocculent slow-growing mycelium on PDA-tet (Fig. $3 \mathrm{E}$ and $\mathrm{F}$ ). With age, a light-brown to dark-brown coloration devel-

Table 3. Incidence of fungal species isolated from olive trees in the main olive-production areas of California

\begin{tabular}{|c|c|c|c|c|c|c|c|c|c|c|c|c|c|c|c|}
\hline \multirow[b]{3}{*}{ District/Co./City* } & & & & \multicolumn{12}{|c|}{ Number $(\%)$ samples yielding } \\
\hline & \multicolumn{3}{|c|}{ Total samples ${ }^{\mathrm{w}}$} & \multicolumn{2}{|c|}{$\begin{array}{l}\text { Botryosphaer- } \\
\text { iaceae }\end{array}$} & \multicolumn{2}{|c|}{$\begin{array}{l}\text { Diaporthe- } \\
\text { Phomopsis }\end{array}$} & \multicolumn{2}{|c|}{ Diatrypaceae } & \multirow{2}{*}{$\begin{array}{c}\begin{array}{c}\text { Basidio- } \\
\text { mycota }\end{array} \\
\text { Ck }\end{array}$} & \multirow{2}{*}{$\begin{array}{c}\begin{array}{c}\text { P.a.- } \\
\text { P.m. }\end{array} \\
\mathrm{Ck}\end{array}$} & \multicolumn{2}{|c|}{$\operatorname{Mix}^{\mathbf{y}}$} & \multicolumn{2}{|c|}{ Others $^{\mathrm{z}}$} \\
\hline & Or & $\mathbf{C k}$ & Td & $\mathbf{C k}$ & Td & $\mathbf{C k}$ & Td & Ck & Td & & & Ck & Td & $\mathbf{C k}$ & Td \\
\hline Sacramento Valley & 22 & 201 & 68 & $99(49.3)$ & $18(26.5)$ & - & - & $26(12.9)$ & - & - & - & $8(4)$ & - & $68(33.8)$ & $50(73.5)$ \\
\hline Butte Co. & 6 & 62 & 11 & $20(32.3)$ & $6(54.5)$ & - & - & $7(11.3)$ & - & - & - & - & - & $35(56.5)$ & $5(45.5)$ \\
\hline Glenn Co. & 6 & 11 & 37 & $5(45.5)$ & $4(10.8)$ & - & - & $6(54.5)$ & - & - & - & - & - & - & $33(89.2)$ \\
\hline Sacramento Co. & 3 & 33 & 10 & $15(45.5)$ & $39(30)$ & - & - & $4(12.1)$ & - & - & - & - & - & $14(42.4)$ & $7(70)$ \\
\hline Sacramento & - & 5 & - & $4(80)$ & - & - & - & - & - & - & - & $1(20)$ & - & - & - \\
\hline Solano Co. & 2 & 25 & - & $10(40)$ & - & - & - & $6(24)$ & - & - & - & $4(16)$ & - & $5(20)$ & - \\
\hline Tehama Co. & 3 & 25 & 10 & $13(52)$ & $5(50)$ & - & - & $3(12)$ & - & - & - & $1(4)$ & - & $8(32)$ & $5(50)$ \\
\hline Yolo Co. & 2 & 28 & - & $22(78.6)$ & - & - & - & - & - & - & - & $2(7.1)$ & - & $4(14.3)$ & - \\
\hline Davis* & - & 12 & - & $10(83.3)$ & - & - & - & - & - & - & - & - & - & $2(16.7)$ & - \\
\hline North Coast & 8 & 107 & 31 & $30(28)$ & $6(19.4)$ & $5(4.7)$ & $9(29)$ & $24(22.4)$ & 7 (22.6) & $18(16.8)$ & $5(4.7)$ & $5(4.7)$ & - & $20(18.7)$ & $9(29)$ \\
\hline Napa Co. & 5 & 28 & 31 & $8(28.6)$ & $6(19.4)$ & $1(3.6)$ & $9(29)$ & $7(25)$ & $7(22.6)$ & $10(35.7)$ & - & $2(7.1)$ & - & - & $9(29)$ \\
\hline Napa $*$ & - & 7 & - & $3(42.9)$ & - & - & - & $2(28.6)$ & - & - & - & $1(14.3)$ & - & $1(14.3)$ & - \\
\hline San Francisco * & - & 10 & - & $4(40)$ & - & $2(20)$ & - & - & - & - & - & & - & $4(40)$ & - \\
\hline Sonoma Co. & 3 & 53 & - & $10(18.9)$ & - & - & - & $13(24.5)$ & - & $8(15.1)$ & $5(9.4)$ & $2(3.8)$ & - & $15(28.3)$ & - \\
\hline Sonoma $*$ & - & 9 & - & $4(44.4)$ & - & $2(22.2)$ & - & $2(22.2)$ & - & - & $1(11.1)$ & & - & - & - \\
\hline San Joaquin Valley & 18 & 207 & 29 & $91(44)$ & $7(24.1)$ & $44(21.3)$ & $3(10.3)$ & - & - & $2(1)$ & - & $25(12.1)$ & $4(13.8)$ & $45(21.7)$ & $15(51.7)$ \\
\hline Fresno Co. & 3 & 24 & 8 & $12(50)$ & $3(37.5)$ & $5(20.8)$ & $2(25)$ & - & - & $2(8.3)$ & - & $3(12.5)$ & $2(25)$ & $2(8.3)$ & $1(12.5)$ \\
\hline Madera Co. & 6 & 71 & - & $35(49.3)$ & - & $19(26.8)$ & - & - & - & - & - & $5(7)$ & - & $12(16.9)$ & - \\
\hline Merced Co. & 5 & 44 & 21 & $15(34.1)$ & $4(19)$ & $3(6.8)$ & $1(4.8)$ & - & - & - & - & $4(9.1)$ & $2(9.5)$ & $22(50)$ & $14(66.7)$ \\
\hline Merced* & - & 13 & - & $6(46.2)$ & - & $3(23.1)$ & - & - & - & - & - & $2(15.4)$ & - & $2(15.4)$ & - \\
\hline Tulare Co. & 4 & 55 & - & $23(41.8)$ & - & $14(25.5)$ & - & - & - & - & - & $11(20)$ & - & $7(12.7)$ & - \\
\hline Central Coast & 7 & 90 & - & $40(44.4)$ & - & $22(24.4)$ & - & $2(2.2)$ & - & - & $7(7.8)$ & $12(13.3)$ & - & $7(7.8)$ & - \\
\hline Santa Barbara Co. & 2 & 28 & - & 22 (78.6) & - & $5(17.9)$ & - & - & - & - & - & $1(3.6)$ & - & - & - \\
\hline Santa Barbara* & - & 4 & - & $3(75)$ & - & $1(25)$ & - & - & - & - & - & & - & - & - \\
\hline Ventura Co. & 5 & 58 & - & $15(25.9)$ & - & $16(27.6)$ & - & $2(3.4)$ & - & - & $7(12.1)$ & $11(19)$ & - & $7(12.1)$ & - \\
\hline Southern California & 4 & 70 & - & $30(42.9)$ & - & $16(22.9)$ & - & $11(15.7)$ & - & - & - & $8(11.4)$ & - & $5(7.1)$ & - \\
\hline Riverside Co. & 4 & 64 & - & $25(39.1)$ & - & $16(25)$ & - & $11(17.5)$ & - & - & - & $7(10.9)$ & - & $5(7.8)$ & - \\
\hline Temecula* & - & 6 & - & $5(83.3)$ & - & - & - & - & - & - & - & $1(16.7)$ & - & - & - \\
\hline Total & 59 & 675 & 128 & $290(43)$ & $31(24.2)$ & 87 (12.9) & $12(9.4)$ & $63(9.3)$ & $7(5.5)$ & $20(3)$ & $12(1.8)$ & $58(8.6)$ & $4(3.1)$ & $145(21.5)$ & $74(57.8)$ \\
\hline
\end{tabular}

w Or. $=$ Total number of orchards surveyed. $\mathrm{Ck} .=$ Perennial canker. Td. $=$ Twig dieback

× P.a. = Phaeomoniella chlamydospora P.m. $=$ Phaeoacremonium aleophilum.

y $\mathrm{Mix}=$ Number of samples from which two or more fungal species were isolated from the same symptom.

$\mathrm{z}$ Others $=$ Number of samples from which different fungi were isolated (endophytes, saprophytes and/or unknown fungi). 


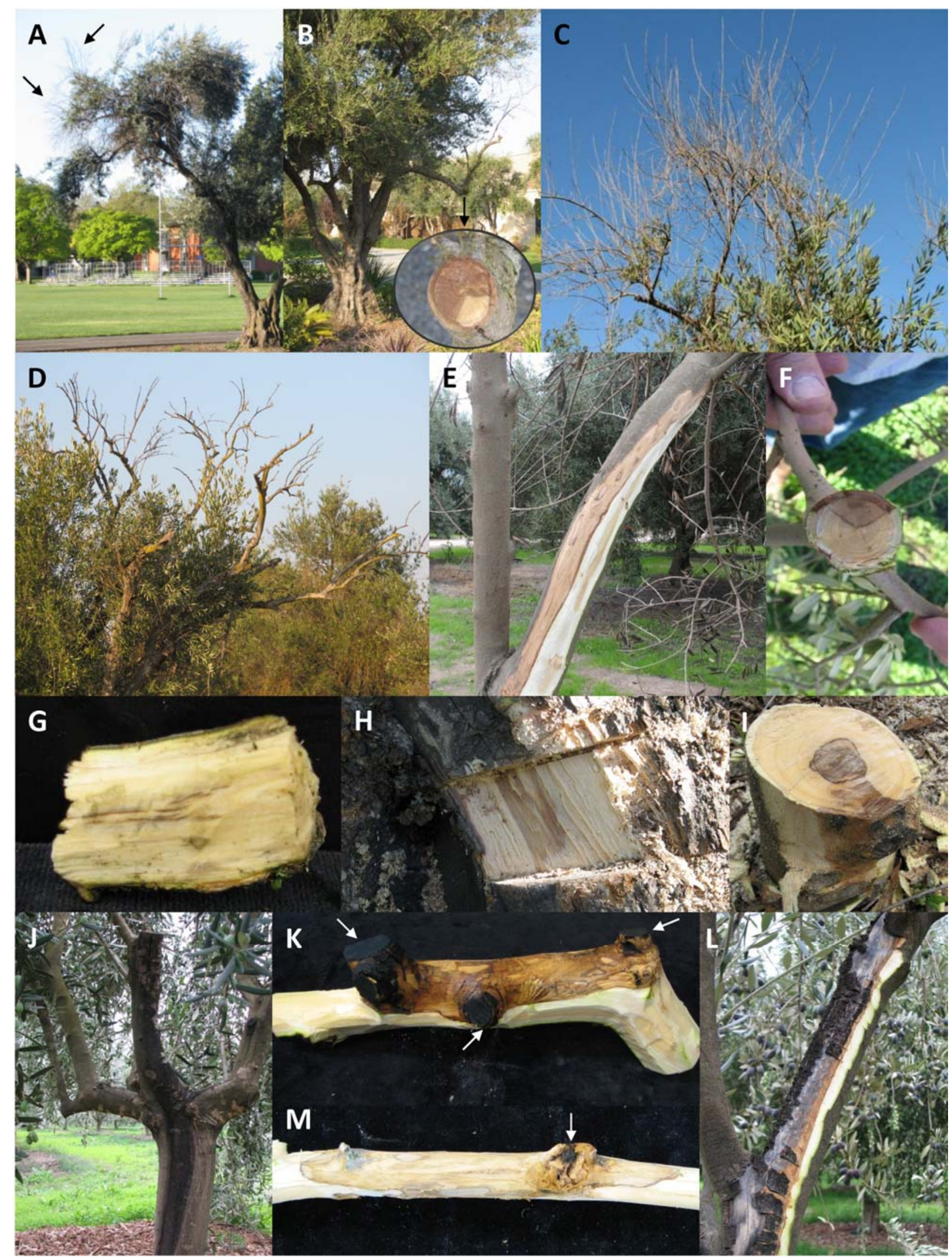

Fig. 2. Olive twig and branch dieback symptoms observed in olives in California orchards. A, Black arrows show moderate symptoms of twig dieback in an ornamental olive tree in the city of Davis. B, Branch dieback in an ornamental tree located in a private garden in the city of Merced. Cross-section of this branch showed a wedge-shaped canker. C, Severe twig dieback observed in a commercial olive orchard. D, Severe branch dieback. E, Perennial canker in an olive branch showing a well-defined dark line of demarcation between infected and healthy tissues. F, Wedge-shaped cankers were the most prevalent vascular symptom observed in symptomatic wood. G, Dark streaking of the wood. H, Light brown symptomatic wood tissue. I, Cankers were also observed at the crown of olive trees. J, Canker observed to develop from a large pruning cut. K, White arrows show several pruning wounds in an olive branch from where perennial canker developed. L, Cankers were also observed to be associated with sunburn areas. M, Cankers were also observed to develop from olive knot wounds caused by the bacterium Pseudomonas syringae pv. savastanoi in both twigs and branches. 


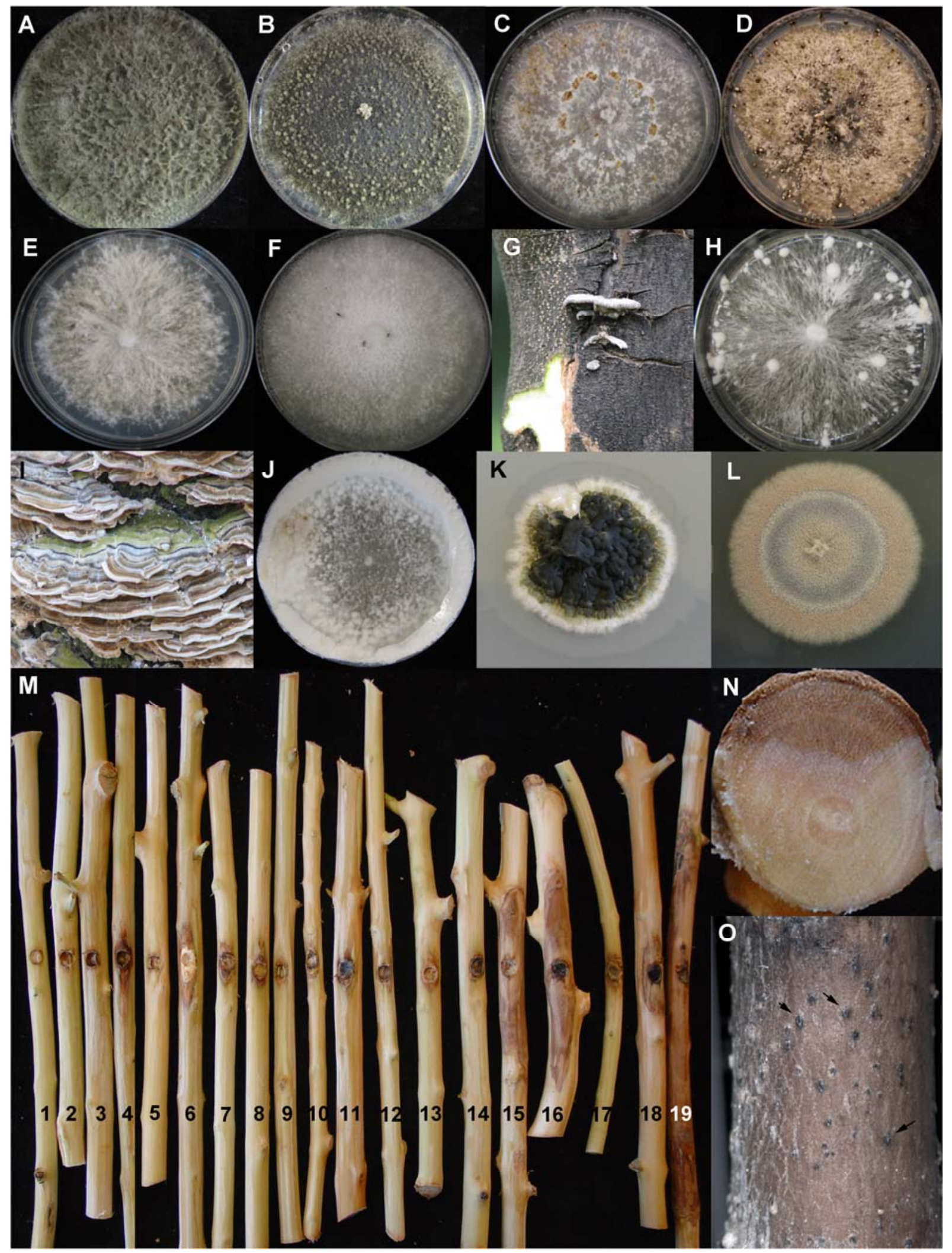

Fig. 3. Four-week-old colony morphology on potato dextrose agar of the different fungal genera isolated from olive trees in California. A, Neofusicoccum mediterraneum. B, Dothiorella iberica. C, Diaporthe viticola. D, Phomopsis sp. group 1. E, Diatrype stigma. F, Eutypa lata. G, Basidiocarps of Schizophyllum commune on an olive tree trunk. H, Schizophyllum commune colony morphology. I, Basidiocarps of Trametes versicolor on an olive tree trunk. J, Trametes versicolor colony morphology. K, Phaeomoniella chlamydospora. L, Phaeoacremonium aleophilum. M, Canker length caused for each of the fugal species identified in this study. 1) control, 2) Diatrype stigma UCD23-Oe, 3) Diatrype oregonensis UCD60-Oe, 4) Eutypa lata UCD143-Oe, 5) Schizophyllum commune UCD296-Oe, 6) Trametes versicolor UCD461-Oe, 7) Diaporthe viticola UCD316Oe, 8) Phomopsis sp. group 1 UCD181-Oe, 9) Phaeomoniella chlamydospora UCD306-Oe, 10) Phaeoacremonium aleophilum UCD468-Oe, 11) Lasiodiplodia theobromae UCD527-Oe, 12) Dothiorella iberica UCD163-Oe, 13) Botryosphaeria dothidea UCD62-Oe, 14) Diplodia seriata UCD340-Oe, 15) Diplodia mutila UCD127-Oe, 16) Diplodia mutila UCD127-Oe, 17) Neofusicoccum vitifusiforme UCD622-Oe, 18) Neofusicoccum luteum UCD369-Oe, and 19) Neofusicoccum mediterraneum UCD1-Oe. N, The same wedge-shaped cankers observed in symptomatic olive wood in the field were reproduced in the pathogenicity test. 0, Black arrows show pycnidia of Neofusicoccum mediterraneum embedded in the bark of a young olive stem. 
oped at the center of the colony on the reverse. Some cultures exhibited slow growth with irregular and lobate colony margins. Conidia produced by pycnidia in the plates were filiform and mostly curved in shape. These characters were consistent with the description of species in the Diatrypaceae family (38). Morphological characteristics allowed us to identify several isolates as Eutypa lata; however, identification to species level for the rest of the isolates was not possible based only on colony and conidia morphology. Diatrypaceous isolates were the third most prevalent fungi isolated from both cankers (9.3\% of the total) and twig dieback (5.5\% of the total) and were primarily found in orchards located throughout the North Coast and the Sacramento Valley (Table 3).

Less common fungi isolated from symptomatic trees were the basidiomycete species Schizophyllum commune Fr. and Trametes versicolor (L.) Lloyd, which were first identified based on the basidiocarps observed on the trees (Fig. 3G and I). Schizophyllum commune basidiocarps were also observed on PDA culture media in vitro (Fig. $3 \mathrm{H}$ ). Additionally, comparison with previously identified isolates from grapevines available in our collection allowed us to identify the slow-growing mycelia of Phaeomoniella chlamydospora Crous \& W. Gams and Phaeoacremonium aleophillum W. Gams, Crous, M.J. Wingfield \& L. Mugnai (Fig. 3K and L), which were sporadically isolated from olive cankers from orchards sampled in both North and Central Coast olive-producing regions (Table 3). Other fungi isolated from both cankers and/or dead twigs were Alternaria alternata (Fr.) Keissl., Alternaria citri Ellis \& N. Pierce, Alternaria sp., Aspergillus sp., Biscogniauxia mediterranea (De Not.) Kuntze, Camarosporium brabeji Marincowitz, M.J. Wingf. \& Crous, Coniozyma leucospermi (Crous \& Denman) Crous, Drechslera erythrospila (Drechsler) Shoemaker, Epicoccum nigrum Link, Nigrospora oryzae (Berk. \& Broome) Petch, Paecilomyces sinensis Q.T. Chen, S.R. Xiao \& Z.Y. Shi, Penicillium cecidicola Seifert, Hoekstra \& Frisvad, Penicillium sp., Phoma macrostoma Mont., and Sordaria sp. These fungi were isolated from 21.5 and $57.8 \%$ of branch cankers and twig dieback (Table 3 ), respectively; however, the occurrence of each species was highly variable. Among them, species of Aspergillus and Alternaria were the most prevalent. Most of these fungi were identified to species level based on molecular identification of the ITS region of rRNA (ITS1-5.8S-ITS2).

Phylogeny. PCR amplifications of the ITS, BT, and EF1- $\alpha$ regions gave products of approximately $0.6,0.4$, and $0.2 \mathrm{~kb}$, respectively. The Botryosphaeriaceae combined ITS, BT, and EF1- $\alpha$ analysis included 40 taxa and contained 1,240 total characters with 847 being constant, 57 parsimony-uninformative, and 339 parsimony-informative. The heuristic search produced 18 most parsimonious trees with 629 steps each $(\mathrm{CI}=0.803, \mathrm{RI}=0.936)$ (trees not shown). The analysis confirmed the identification of eight different species in the Botryosphaeriaceae from California olive trees, including Lasiodiplodia theobromae, Diplodia mutila, Diplodia seriata, Dothiorella iberica, Botryosphaeria dothidea, Neofusicoccum mediterraneum, Neofusicoccum vitifusiforme, and Neofusicoccum luteum. Botryosphaeriaceous isolates from olives from California grouped in well-supported clades ( $>90 \%$ bootstrap value) along with ex-type specimens retrieved from GenBank when available and/or with previously identified species in the Botryosphaeriaceae from California from different hosts.

The combined ITS and BT analysis of Diatrypaceae spp., $P h$. chlamydospora and Pm. aleophilum included 34 taxa and contained 940 total characters with 473 being constant, 52 parsimonyuninformative, and 415 parsimony-informative. The heuristic search produced 24 most parsimonious trees with 1,024 steps each $(\mathrm{CI}=0.709, \mathrm{RI}=0.866)$ (trees not shown). The analysis confirmed the identification of three species of Diatrypaceae, Diatrype oregonensis, Diatrype stigma, and Eutypa lata, and corroborated the identification of both Ph. chlamydospora and Pm. aleophilum from olive in California.

The combined ITS and EF1- $\alpha$ analysis of Diaporthe and Phomopsis spp. included 30 taxa and contained 883 total charac- ters with 519 being constant, 47 parsimony-uninformative, and 317 parsimony-informative. The heuristic search produced 4 most parsimonious trees with 1,001 steps each $(\mathrm{CI}=0.596, \mathrm{RI}=0.771)$ (trees not shown). The analysis confirmed the identification of three different species, including Diaporthe viticola and two nondetermined Phomopsis spp., namely, Phomopsis sp. group 1 and Phomopsis sp. group 2. The combined ITS and EF1- $\alpha$ analysis showed isolates UCD316-Oe and 327-Oe to group in a well-supported clade (100\% bootstrap) with different Diaporthe viticola isolates from $V$. vinifera, including the ex-type isolate CBS 113201. California olive isolates UCD181-Oe, UCD-182-Oe, and UCD213-Oe, and UCD233-Oe, UCD237-Oe, UCD248-Oe, UCD278-Oe, and UCD580-Oe grouped in two separate well-supported clades (100\% bootstrap) and shared identical gene sequences with Phomopsis sp. isolates UCD1685S1 and CAL-5 from $V$ vinifera from California, respectively.

California olive isolates UCD296-Oe, UCD297-Oe, UCD298Oe, and UCD304-Oe and isolates UCD311-O3, UCD312-Oe, UCD313-Oe, and UCD461-Oe shared 99 and 100\% similarity with most ITS sequences of Schizophyllum commune and Trametes versicolor from GenBank, respectively. Figure 4 shows the ITS phylogenetic analysis with all fungal taxa used in this study, including those identified from olive trees in California.

Pathogenicity test. Mean lengths of vascular discoloration caused by the 18 fungal isolates tested and the control in the Manzanillo and Sevillano olive cultivars are shown in Table 4. Six months after inoculation, cankers observed in cross-section from the inoculated branches were very similar in shape (wedge-shaped to round) to those observed and collected from commercial olive orchards (Fig. 3M and N). Canker shape caused by all inoculated species was very similar with the exception of Pa. chlamydospora and $P h$. aleophilum, which caused a necrotic streaking of the vascular system. Although all fungal species tested in the pathogenicity test caused cankers and/or vascular discoloration of the wood significantly different in length $(P<0.05)$ than the control, virulence varied among species (Table 4$)$. The botryosphaeriaceous taxa N. mediterraneum and Diplodia mutila were shown to be the most virulent species in both Manzanillo (63.1 and $43.6 \mathrm{~mm}$ mean lesion, respectively) and Sevillano (74.8 and $76 \mathrm{~mm}$ mean lesion, respectively) cultivars (Table 4). N. vitifusiforme and T. versicolor in both cultivars and E. lata in the Sevillano cultivar followed in virulence, causing mean lesions more than $10 \mathrm{~mm}$ in length (Table 4). The remaining fungal species caused mean lesions that were between 5 and $10 \mathrm{~mm}$ in length. Diatrype stigma and D. oregonensis were the least virulent species, causing mean lesions less than $4 \mathrm{~mm}$ in length (Table 4). Percentages of fungal recovery were high $(>70 \%)$ for all treatments with the exception of Pa. chlamydospora, D. stigma, and D. oregonensis $(50 \%$ or lower) (Table 4). Both Manzanillo and Sevillano olive cultivars used in the pathogenicity test were susceptible to infection by all fungal species tested. However, ANOVA revealed that Sevillano was more susceptible to infection caused by $N$. mediterraneum, Diplodia mutila, E. lata, and Diaporthe viticola than Manzanillo. On the other hand, Manzanillo was more susceptible to infection caused by $L$. theobromae and Pa. chlamydospora than Sevillano (Table 4).

\section{Discussion}

Eighteen fungal species belonging to 12 different genera within eight different families were isolated from symptomatic wood of olive trees affected by twig and branch dieback in California and identified by means of morphological characters and multigene sequence analyses. These species included Botryosphaeria dothidea, Diaporthe viticola, Diatrype oregonensis, Diatrype stigma, Diplodia mutila, Diplodia seriata, Dothiorella iberica, Eutypa lata, Lasiodiplodia theobromae, Neofusicoccum luteum, Neofusicoccum mediterraneum, Neofusicoccum vitifusiforme, Phaeoacremonium aleophilum, Phaeomoniella chlamydospora, Phomopsis sp. group 1, Phomopsis sp. group 2, Schizophyllum commune, and Trametes versicolor. 


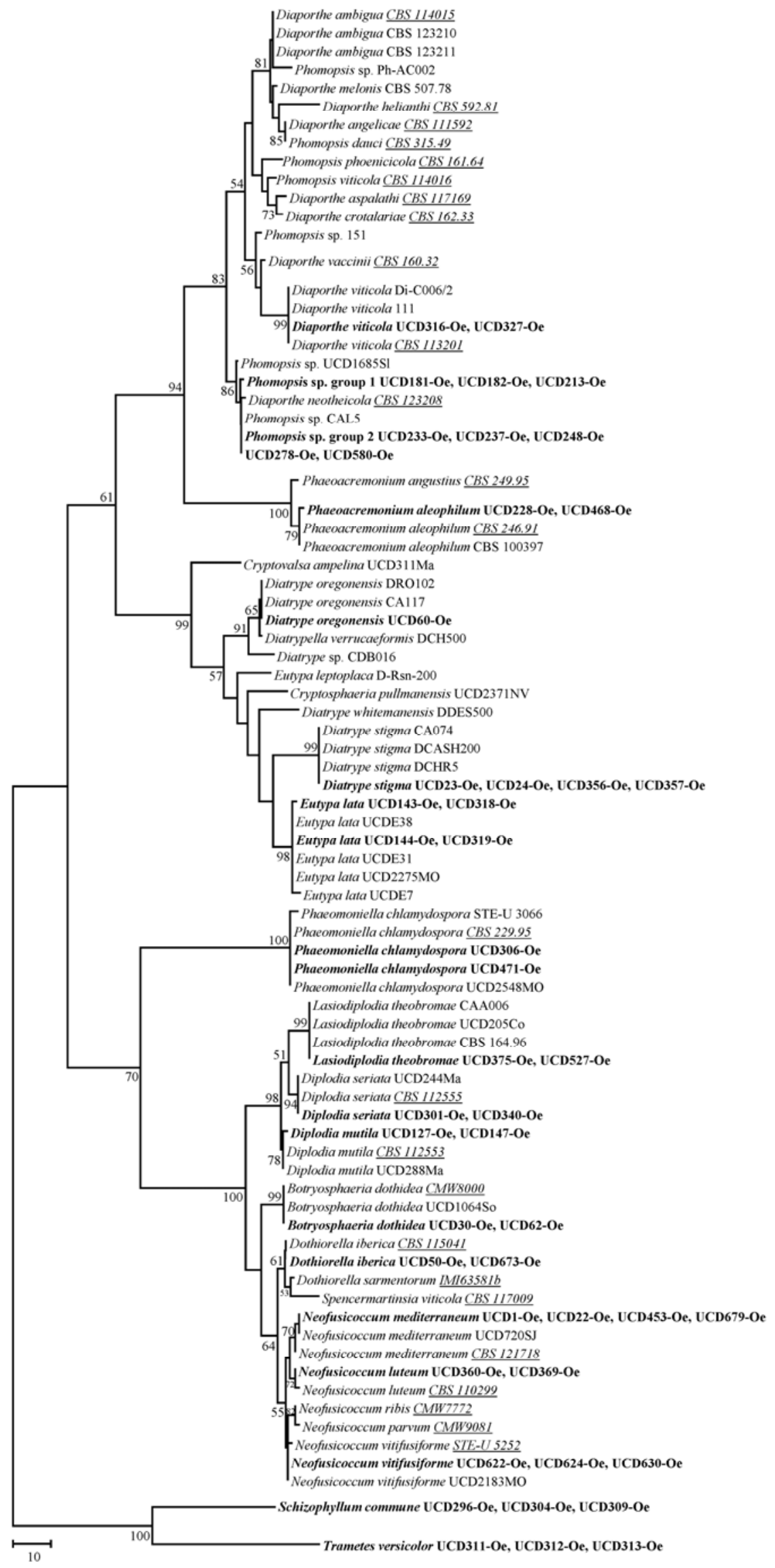

Fig. 4. One of the 62 most parsimonious trees representing all taxa used in this study obtained from the internal transcribed spacer (ITS) sequence data (length $=550, \mathrm{Cl}=$ $0.552920, \mathrm{RI}=0.927213$, and the composite index $=0.514182$ ). Bootstrap support values higher than $50 \%$ from 1,000 replications are shown at the nodes. Bold isolates represent isolates from olive from California. Italics and underline isolates represent the ex-type specimen. 
Olive twig and branch dieback symptoms were manifested by defoliation and/or wilting of the leaves in young twigs. Although these symptoms were typically observed to affect only part of the tree, entire trees were occasionally observed to be affected in some of the orchards surveyed. In the field, these symptoms can easily be confused with those resembling Verticillium wilt $(13,26)$, which could explain to some extent why olive twig and branch dieback has remained overlooked and consequently little studied by plant pathologists for many years. Nevertheless, olive branch and twig dieback can be distinguished from Verticillium wilt by the presence of flat to slightly sunken and darker areas along the affected stems, older branches, and/or trunk, which reveal perennial cankers when the bark is removed. Additionally, pycnidia and/or perithecia of some of the fungi associated with olive twig and branch dieback can be found embedded in the bark of the affected areas. Moreover, cankered zones along branches also reveal a well-defined dark line of demarcation between infected and healthy tissues. The dark streaking of the wood symptom observed in olive trees in this study, on the other hand, and from which Pa. chlamydospora and Pm. aleophilum were isolated, resembles the vascular symptoms associated with Verticillium wilt, caused by Verticillium dahliae. In this case, sample collection and fungal identification by either traditional plating and/or molecular techniques would be required in order to properly identify the causal agent.

Perennial cankers and subsequent dieback have been widely studied in many different perennial hosts worldwide. However, to date, only a few studies regarding this problem have been carried out on olive trees $(20,25-27,32)$. Additionally, most of these studies have focused their research only in a single fungal species as the cause of olive dieback $(25-27,32)$. The current study represents the first attempt to elucidate the etiology of olive twig and branch dieback, and contrary to previous studies, it shows that not only one but a complex of several taxonomically unrelated fungal species are involved in this disease.

Among all the fungal taxa isolated from symptomatic wood of olive trees in this study, species of Botryosphaeriaceae were the most prevalent. Botryosphaeriaceae species are primarily known to cause olive fruit rot $(6,15,19,20,22)$, including the dalmatian disease, caused by $B$. dothidea and considered the most important fruit disease of table olives (20). However, several botryosphaeriaceous taxa have also been reported to cause olive branch dieback, including $N$. luteum in New Zealand (32), N. ribis and $N$. mediterraneum in Spain $(20,25)$, and Diplodia seriata in Croatia (14). Most recently, Moral et al. (20) reported B. dothidea, D. seriata, and $N$. mediterraneum to cause branch cankers and blighted shoots of olive trees in California. The current study corroborates the presence of these latter species in olive trees in California and adds five new records of Botryosphaeriaceae species in olive trees in America, including Diplodia mutila, Dothiorella iberica, L. theobromae, N. luteum, and N. vitifusiforme. Additionally, this study reports for the first time $D$. mutila, $D$. iberica, and $L$. theobromae to occur in olive trees. Moreover, complementary to the study conducted by Moral et al. (20) in which only three counties (Glenn, Madera, and Fresno) were surveyed in California, the present study shows Botryosphaeriaceae species to be widespread throughout all olive-growing regions in the state, including ornamental olive trees planted along streets and in both public and private gardens in several California cities. The pathogenicity trial showed the Botryosphaeriaceae species, $N$. mediterraneum and $D$. mutila, to be the most virulent in olive trees among all the fungi in this study. The high virulence of $N$. mediterraneum showed in this study agrees with the results reported by Moral et al. (20) in which this species was shown to be very aggressive when inoculated into olive branches. However, the current study shows that $D$. mutila can be equally or more virulent than $N$. mediterraneum in olive trees. On the other hand, the rest of Botryosphaeriaceae species tested in this study were significantly less virulent, with $D$. seriata and $D$. iberica as the least virulent of the Botryosphaeriaceae. Similarly, B. dothidea and D. seriata isolates from California have been reported to be either nonpathogenic or weakly virulent when inoculated in olive branches (20) or grapevines (45). The family Botryosphaeriaceae includes several well-known plant pathogens that cause perennial cankers and consequent dieback in a broad range of economically important woody perennial crops and ornamental plants, as well as in both native and introduced forest tree species $(8,43)$. In California, species of Botryosphaeriaceae not only occur in a wide range of different hosts (4) but are also considered one of the major threats to the almond (12), grapevine (43), and pistachio (17) industries, reducing yields and shortening the life span of these crops. The present study adds olive trees as a major host of Botryosphaeriaceae species in

Table 4. Mean lesion length caused by different fungal species isolated from diseased olive trees from California on 2- and/or 3-year-old branches of Manzanillo and Sevillano olive cultivars 6 months after inoculation

\begin{tabular}{|c|c|c|c|c|c|c|c|}
\hline \multirow[b]{2}{*}{ Species } & \multirow[b]{2}{*}{ Isolate } & \multicolumn{3}{|c|}{ Manzanillo } & \multicolumn{3}{|c|}{ Sevillano } \\
\hline & & Lesion length $^{\mathrm{x}} \pm \mathrm{SE}$ & Signif. dif. ${ }^{y}$ & Samples ${ }^{\mathrm{z}}$ & Lesion length $\pm \mathrm{SE}$ & Signif. dif. & Samples \\
\hline Neofusicoccum mediterraneum & UCD1-Oe & $63.1 \pm 6.6 \mathrm{a}$ & A & 10 & $74.8 \pm 5.2 \mathrm{a}$ & B & 10 \\
\hline Diplodia mutila & UCD127-Oe & $43.6 \pm 6.4 \mathrm{~b}$ & A & 10 & $76.0 \pm 6.6 \mathrm{a}$ & B & 9 \\
\hline Neofusicoccum vitifusiforme & UCD622-Oe & $12.4 \pm 1.5 \mathrm{c}$ & A & 10 & $10.5 \pm 0.8 b$ & A & 10 \\
\hline Ttrametes versicolor & UCD461-Oe & $10.1 \pm 0.7 \mathrm{~d}$ & A & 9 & $10.3 \pm 1.2 \mathrm{bc}$ & A & 10 \\
\hline Neofusicoccum luteum & UCD369-Oe & $9.0 \pm 0.9 \mathrm{de}$ & A & 10 & $7.5 \pm 1.1 \mathrm{def}$ & A & 10 \\
\hline Eutypa lata & UCD143-Oe & $8.4 \pm 0.3 \mathrm{ef}$ & A & 10 & $10.1 \pm 0.9 \mathrm{bcd}$ & B & 9 \\
\hline Botryosphaeria dothidea & UCD62-Oe & $8.1 \pm 0.8 \mathrm{ef}$ & A & 10 & $9.0 \pm 1.0 \mathrm{cde}$ & A & 10 \\
\hline Lasiodiploida theobromae & UCD527-Oe & $7.9 \pm 0.7 \mathrm{efg}$ & A & 10 & $6.1 \pm 0.4 \mathrm{efg}$ & B & 9 \\
\hline Phomopsis sp. group 2 & UCD248-Oe & $7.7 \pm 0.5$ efg & A & 10 & $7.3 \pm 0.6 \mathrm{def}$ & A & 10 \\
\hline Diaporthe viticola & UCD316-Oe & $7.2 \pm 0.5 \mathrm{fgh}$ & A & 10 & $9.4 \pm 0.7$ cde & B & 9 \\
\hline Diplodia seriata & UCD340-Oe & $7.2 \pm 0.4 \mathrm{fgh}$ & A & 9 & $8.1 \pm 0.8 \mathrm{def}$ & A & 10 \\
\hline Phomopsis sp. group 1 & UCD181-Oe & $6.7 \pm 0.7 \mathrm{gh}$ & A & 9 & $6.5 \pm 0.6 \mathrm{fg}$ & A & 8 \\
\hline Dothiorella iberica & UCD163-Oe & $6.1 \pm 0.5 \mathrm{hi}$ & A & 7 & $4.3 \pm 0.7 \mathrm{hi}$ & A & 9 \\
\hline Phaeomoniella chlamydospora & UCD306-Oe & $6.0 \pm 0.6 \mathrm{hi}$ & A & 4 & $3.1 \pm 0.4 \mathrm{ij}$ & B & 5 \\
\hline Schizophyllum commune & UCD296-Oe & $5.5 \pm 0.6 \mathrm{ij}$ & A & 10 & $6.3 \pm 0.8 \mathrm{gh}$ & A & 8 \\
\hline Phaeoacremonium aleophilum & UCD468-Oe & $5.1 \pm 0.6 \mathrm{ij}$ & A & 8 & $4.9 \pm 0.6 \mathrm{hi}$ & A & 8 \\
\hline Diatrype stigma & UCD23-Oe & $3.2 \pm 0.2 \mathrm{k}$ & A & 5 & $4.3 \pm 0.8 \mathrm{hi}$ & A & 4 \\
\hline Diatrype oregonensis & UCD60-Oe & $2.9 \pm 0.6 \mathrm{k}$ & A & 3 & $3.3 \pm 0.3 \mathrm{ij}$ & A & 5 \\
\hline Control & & $1.4 \pm 0.11$ & A & & $1.3 \pm 0.1 \mathrm{k}$ & A & \\
\hline
\end{tabular}

${ }^{x}$ Values represent the average in millimeters of acropetal and basipetal extent of vascular discoloration (10 repetitions per isolate) measured from the point of inoculation. SE = Standard error of the mean. Means with the same letter are not significantly different at the 0.05 level.

${ }^{y}$ Significant differences of each isolate among Manzanillo and Sevillano cultivars. Means with the same capital letters in each row are not significantly different at the 0.05 level.

${ }^{\mathrm{z}}$ Number of samples from which the fungus was re-isolated out of 10 samples inoculated. 
California and highlights the important role that several of these species play on twig and olive branch dieback disease.

Fungal taxa in the genus Phomopsis (teleomorph: Diaporthe Nitschke) were the second most prevalent fungi isolated from olive cankers and twig dieback in California. The genus Phomopsis contains over 900 species, which are known to be cosmopolitan and found as endophytes, parasites, and saprotrophs in a wide range of hosts $(8,40)$. However, no record of either the asexual (Phomopsis) or the sexual form (Diaporthe) has been reported to occur in olive trees (8). Two different Phomopsis spp. were isolated from olive wood in California, which represents the first report of olive trees as host of species belonging to the Phomopsis genus. Although combined ITS and EF1- $\alpha$ phylogenetic analyses showed Phomopsis isolates from olive in California to share 99\% homology with previously identified Phomopsis sp. isolates UCD1685S1 (46) and CAL-5 (29) from V. vinifera in California, they differed from the rest of Phomopsis and/or Diaporthe species available in GenBank; and thus, they were designated as Phomopsis sp. group 1 and Phomopsis sp. group 2. These results indicate both Phomopsis spp. isolated from olive and grapevine in California are potential novel species within this genus; however, further morphological and phylogenetic studies, which were beyond the scope of this study, are currently underway to confirm whether or not these two taxa represent novel Phomopsis spp. To date, D. viticola has only been reported to occur on Hydrangea macrophylla and Vitis vinifera, and its distribution is restricted to Germany and Portugal $(8,28,40)$. Diaporthe viticola is reported for the first time here to occur in olive trees, as well as in a country other than Germany and Portugal. Phomopsis sp. group 1, Phomopsis sp. group 2, and D. viticola were shown to be pathogenic in olive branches; however, all three species were considered to be intermediately virulent based on the extent of vascular wood death they caused.

The diatrypaceous fungi $E$. lata, D. oregonensis, and D. stigma were the third most prevalent fungi isolated from olive cankers in California. To date, E. lata, D. oregonensis, and D. stigma have been shown to occur in 136, 114, and 10 different plant hosts, respectively (8). However, only $E$. lata has been previously reported in olive trees causing olive dieback in Greece (27) and in Italy (35). Therefore, this is the first report of E. lata and D. oregonensis and D. stigma in olive trees in America and worldwide, respectively. Pathogenicity of E. lata in olive trees was demonstrated by Rumbos (27) in the early 1990s, who reported Greek isolates of this species to be highly virulent, causing up to $52 \mathrm{~mm}$ canker length 12 months after inoculation. On the other hand, this study showed E. lata isolates from California to be moderately virulent when inoculated in olive branches, causing an average length necrosis significantly less severe. The lower virulence observed with California isolates of E. lata in olive trees could be due to variability in isolate virulence, type of inoculated tissue, shorter incubation period, age of the host, and/or differences in cultivar susceptibility among many other possible factors. However, whether any of these factors play a direct role in the virulence of E. lata in olive remains unclear at this time; and thus, a more complete pathogenicity study using a higher number of both $E$. lata isolates and olive cultivars will be required to further clarify these hypotheses. Contrary to E. lata, D. stigma and D. oregonensis were shown in this study to be the least virulent fungi in olive trees among all fungal species tested. Trouillas and Gubler (37) recently showed grapevine isolates of $D$. stigma and D. oregonensis to cause lesions in grapevines that were not significantly different from those observed in the negative controls, suggesting that these fungi may be saprotrophic on this host. The low virulence of both $D$. stigma and $D$. oregonensis observed in this study supports the hypothesis that these diatrypaceous fungi may act as saprotrophs in olive trees.

Although less prevalent, the basidiomycetous fungi $S$. commune and $T$. versicolor, as well as the grape measles (esca) fungi, $P a$. chlamydospora and Pm. aleophilum, were also sporadically isolated from symptomatic wood of olive trees in California. Among these fungi, T. versicolor and Pm. aleophilum have been previously reported to occur in olive trees in California (1) and in Italy (11), respectively. However, Koch's postulates were not fulfilled. Although moderate and low virulence were shown in this study for $T$. versicolor and Pm. aleophilum, respectively, these pathogenicity tests indicated that both fungi can infect and colonize green live olive tissue. S. commune and Pa. chlamydospora, on the other hand, are reported for the first time here as weak pathogens of olive trees. Additionally, for the first time, this study shows $P a$. chlamydospora to occur in a host other than $V$. vinifera.

All fungal species identified in this study from olive trees, except the two Phomopsis spp., have been widely recognized as important pathogens of grapevines, involved in what is known as the grapevine trunk disease complex worldwide $(21,23,37,45,48,49)$. Results from the field survey conducted in this study showed all these fungi occur in olive orchards either near or adjacent to vineyards throughout California. E. lata, for example, was only isolated from symptomatic olive trees in Napa, Sonoma, and Sacramento counties, which are known to be the grapevine-growing regions with the highest rate of Eutypa dieback of grapevines in California (46). Similarly, the botryosphaeriaceous fungi L. theobromae, $N$. luteum, and $N$. mediterraneum, which have previously been reported to occur in grapevines only in counties throughout the San Joaquin Valley and Southern California $(46,47)$, were primarily isolated from olive trees from the same geographical regions. Another example is D. viticola, a fungal species found almost exclusively on grapevines and associated with Phomopsis cane and leaf spot symptoms (49). In this study, D. viticola was only isolated from olive trees in the grape-growing regions of Napa and Sonoma counties where Phomopsis cane and leaf spot disease is commonly observed on grapevines. However, most of the fungal species isolated from olive cankers and known to be highly virulent on grapevines (E. lata, B. dothidea, L. theobromae, N. luteum, Pa. chlamydospora, and Pm. aleophilum) were found to be of intermediate to low virulence when inoculated in olive branches. These results suggest these fungi are better adapted to infect grapevine wood, and possibly only the most virulent isolates of some of these species would be more aggressive in olive trees. Moral et al. (20) showed isolates of $B$. dothidea from infected olive fruit to be nonpathogenic when inoculated on olive branches and thus specialized in which olive tissues are infected. A more thorough pathogenicity study in olive trees using multiple high virulence isolates of each fungal species from grapevines and/or other hosts will be required to confirm this hypothesis.

Likewise, several of the botryosphaeriaceous fungi isolated from olive trees in this study are also known to cause cankers and consequent dieback in other economically important perennial crops in California. For instance, $B$. dothidea causes panicle and shoot blight of pistachio (17). Moreover, $B$. dothidea, D. seriata, and $N$. mediterraneum cause cankers and dieback of almond (12). Additionally, $N$. mediterraneum was reported to be highly virulent in walnut trees, causing twig and branch dieback (39). In this study, $B$. dothidea and $N$. mediterraneum were mainly found to occur in olive orchards in both Sacramento (Butte, Glenn, Tehama, and Yolo counties) and San Joaquin valleys (Fresno, Madera, Merced, and Tulare counties), which correspond with over 52, 43, and $42 \%$ of the total almond, pistachio, and walnut acres planted in California, respectively (42). Similar to what occurs with grapevines in counties such as Napa and Sonoma, olive trees are often planted near or adjacent to almond, pistachio, and/or walnut orchards throughout both the Sacramento and San Joaquin valleys. This perceived cross infection of multiple hosts by these fungi has been reported to occur in southern Spain, where pistachio and olive trees grow together, sometimes within the same orchard (20), and further support the hypothesis that infected hosts adjacent to olive orchards may serve as sources of inoculum for these pathogens and vice versa (12). Furthermore, in the case of botryosphaeriaceous species, such a proximity of olive trees to almonds, grapevines, pistachios, and/or walnuts throughout these particular growing regions in California and/or other countries could be essential for cross infections, since dissemination of pycnidiospores is primarily 
water-splashed over relatively short distances $(3,44)$. Consequently, larger buffer zones between olive orchards and these crops may reduce risk of cross infection by botryosphaeriaceous species and/or other fungi with a similar mode of spread. However, increasing zones may not be an economically viable alternative for mature and already well-established olive orchards, since removal of old trees would be necessary; but larger buffer zones could be considered when planting new olive orchards in California.

This study has shown significant information regarding the etiology and importance of olive twig and branch dieback as well as the distribution of the causal agents throughout California. However, it has only focused on the status of this disease in traditional mature and low-density olive orchards throughout the state (200 to 500 trees per ha). Traditional olive orchards for the production of either table olives or olive oil are on the other hand no longer popular when establishing new olive plantings worldwide due to the high cost, primarily as a consequence of labor expenses for harvesting and pruning (36). Consequently, olive growers in California are currently switching to super-high-density olive production farming, characterized by high tree density (1,500 to 2,500 trees per ha) and based on hedgerows, machine harvest, and mechanical pruning (36). Severe mechanical pruning will probably be necessary to preserve low tree size in order to maximize machine harvesting efficiency (36). Mechanical pruning in such a high-density crop will create hundreds of thousands of new pruning wounds every year, which are known to be the main point of entrance for all the fungi identified in this study and responsible for causing olive twig and branch dieback $(21,37,43,48)$. Moreover, mechanical harvest may also result in many injuries to the trees which could be susceptible to infection. Therefore, olive twig and branch dieback incidence is suggested to be higher in super-high-density olive orchards than in traditional plantings, in which pruning is sometimes kept to a minimum. Consequently, research needs to be continued, not only to determine the importance of olive twig and branch dieback in this new olive production system, but also to develop and implement effective management strategies under this new approach to farming olives.

\section{Acknowledgments}

This research was partially funded by grants from the California Olive Board. We thank University of California Cooperative Extension Farm Advisors J. H. Connell (UCCE Butte County), C. A. Ingles (UCCE Sacramento County), D. Doll (UCCE Merced County), N. V. O'Connell (UCCE Tulare County), and B. Faber (UCCE Ventura County) for their valuable assistance and guidance in accomplishing the field collections.

\section{Literature Cited}

1. Adaskaveg, J. E., and Ogawa, J. M. 1990. Wood decay pathology of fruit and nut trees in California. Plant Dis. 74:341-352.

2. Agrios, G. N. 2005. Plant Pathology, 5th ed. Elsevier-Academic Press, San Diego, CA.

3. Ahimera, N., Gisler, S., Morgan, D. P., and Michailides, T. J. 2004. Effects of single-drop impactions and natural and simulated rains on the dispersal of Botryosphaeria dothidea conidia. Phytopathology 94:1189-1197.

4. Brooks, F. E., and Ferrin, D. M. 1994. Branch dieback of southern California chaparral vegetation caused by Botryosphaeria dothidea. Phytopathology 84:78-83.

5. Carbone, I., Anderson, J. B., and Kohn, L. M. 1999. A method for designing primer sets for the speciation studies in filamentous ascomycetes. Mycologia 91:553-556

6. Chattaoui, M., Rhouma, A., Krid, S., Ali Triki, M., Moral, J., Msallem, M., and Trapero, A. 2011. First report of fruit rot of olives caused by Botryosphaeria dothidea in Tunisia. Plant Dis. 95:770.

7. Connell, J. H. 2005. History and scope of the olive industry. Pages 1-10 in: Olive Production Manual, 2nd ed. G. S. Sibbet and L. Ferguson, eds. University of California Agricultural and Natural Resources, Publ. 3353, Oakland, CA.

8. Farr, D. F., and Rossman, A. Y. 2012. Fungal Databases, Systematic Mycology and Microbiology Laboratory, ARS, USDA. Retrieved 15 February 2012 from http://nt.ars-grin.gov/fungaldatabases/fungushost/fungushost.cfm

9. Food and Agriculture Organization of the United Nations (FAO). FAOSTAT (C) FAO Statistics Division. Retrieved 15 January 2012 from http://faostat. fao.org/default.aspx

10. Glass Louise, N., and Donaldson, G. C. 1995. Development of primer sets designed for use with the PCR to amplify conserved genes from filamentous Ascomycetes. Appl. Environ. Microbiol. 61:1323-1330.
11. Groenewald, M., Kang, J. C., Crous, P. W., and Gams, W. 2001. ITS and beta-tubulin phylogeny of Phaeoacremonium and Phaeomoniella. Mycol. Res. 105:651-657.

12. Inderbitzin, P., Bostock, R. M., Trouillas, F. P., and Michailides, T. J. 2010. A six locus phylogeny reveals high species diversity in Botryosphaeriaceae from California almond. Mycologia 102:1350-1368.

13. Jiménez-Díaz, R. M., Cirulli, M., Bubici, G., Jiménez-Gasco, M. M., Antoniou, P. P., and Eleftherios, C. T. 2012. Verticillium wilt, a major threat to olive production: Current status and future prospects for its management. Plant Dis. 96:304-329.

14. Kaliterna, K., Ivic, I., Bencic, D., and Mesic, A. 2012. Fisrt report of Diplodia seriata as causal agent of olive dieback in Croatia. Plant Dis. 96:290.

15. Lazzizera, C., Frisullo, S., Alves, A., and Phillips, A. J. L. 2008. Morphology, phylogeny and pathogenicity of Botryosphaeria and Neofusicoccum species associated with drupe rot of olive in southern Italy. Plant Pathol. 57:948-956.

16. Malathrakis, N. E. 1979. Studies on a disease of olive due to fungus Phoma incompta Sacc. \& Mart. Ph.D. diss. University of Athens, Greece.

17. Michailides, T. J. 1991. Pathogenicity, distribution, sources of inoculum, and infection courts of Botryosphaeria dothidea on pistachio. Phytopathology 81:566-573.

18. Molina de la Rosa, J. L., Jiménez Herrera, B., Ruiz Coleto, F., García Zamorano, F., Cano Rodríguez, J., and Pérez García, J. 2004. Técnicas de cultivo: Plagas y enfermedades del olivo. Viceconsejería, Servicio de Publicaciones y Divulgación, eds. Junta de Andalucía, Consejería de Agricultura y Pesca, Sevilla, Spain.

19. Moral, J., Luque, F., and Trapero, A. 2008. First report of Diplodia seriata, the anamorph of "Botryosphaeria" obtusa, causing fruit rot of olive in Spain. Plant Dis. 92:311.

20. Moral, J., Muñoz-Díez, C., González, N., Trapero, A., and Michailides, T. 2010. Characterization and pathogenicity of Botryosphaeriaceae species collected from olive and other hosts in Spain and California. Phytopathology 100:1340-1351

21. Mugnai, L., Graniti, A., and Surico, G. 1999. Esca (black measles) and brown wood-streaking: Two old and elusive diseases of grapevines. Plant Dis. 83:404-418.

22. Phillips, A. J. L., Rumbos, I. C., Alves, A., and Correia, A. 2005. Morphology and phylogeny of Botryosphaeria dothidea causing fruit rot of olives. Mycopathologia 159:433-439.

23. Reisenzein, H., Berger, R., and Nieder, G. 2000. Esca in Austria. Phytopathol. Mediterr. 39:26-34.

24. Rhouma, A., Triki, M. A., Krid, S., and Masallem, M. 2010. First report of a Branch dieback of olive trees in Tunisia caused by a Phoma sp. Plant Dis. 94:636

25. Romero, M. A., Sánchez, M. E., and Trapero, A. 2005. First report of Botryosphaeria ribis as a branch dieback pathogen of olive trees in Spain. Plant Dis. 89:208.

26. Rumbos, I. C. 1988. Cytospora oleina causing canker and dieback of olive in Greece. Plant Pathol. 37:441-444.

27. Rumbos, I. C. 1993. Dieback symptoms on olive trees caused by the fungus Eutypa lata. Bull. OEPP/EPPO Bull. 23:441-445.

28. Santos, J. M., Correia, V. G., and Phillips, A. J. L. 2010. Primers for matingtype diagnosis in Diaporthe and Phomopsis: Their use in teleomorph induction in vitro and biological species definition. Fungal Biol. 114:255-270.

29. Schilder, A. M. C., Erincik, O., Castlebury, L., Rossman, A., and Ellis, M. A. 2005. Characterization of Phomopsis spp. infecting grapevines in the Great Lakes region of North America. Plant Dis. 89:755-762.

30. Schroth, M. N., and Osgood, J. W. 1971. Relationship between the olive knot disease and reduced yield. Calif. Olive Ind. News 25, no. 1 (February).

31. Swofford, D. L. 1999. PAUP*. Phylogenetic Analysis Using Parsimony (*and other methods), version 4.0b4a. Sinauer Associates, Sunderland, MA.

32. Taylor, R. K., Hale, C. N., and Hartill, W. F. T. 2001. A stem canker disease of olive (Olea europaea) in New Zealand. N.Z. J. Crop Hortic. 29:219-228.

33. Teviotdale, B. L. 2005. Diseases of olive. Pages 119-121 in: Olive Production Manual, 2nd ed. G. S. Sibbett and L. Ferguson. eds. University of California Agricultural and Natural Resources, Publ. 3353, Oakland, CA.

34. Thompson, J. D., Higgins, D. G., and Gibson, T. J. 1994. CLUSTAL W: Improving the sensitivity of progressive multiple sequence alignment through sequence weighting, position-specific gap penalties and weight matrix choice. Nucleic Acids Res. 22:4673-4680.

35. Tosi, L., and Natalini, G. 2009. First report of Eutypa lata causing dieback of olive trees in Italy. Plant Pathol. 58:398

36. Tous, J., Romero, A., Hermoso, J. F., and Ninot, A. 2011. Mediterranean clonal selections evaluated for modern hedgerow olive oil production in Spain. Calif. Agric. 65:34-40.

37. Trouillas, F. P., and Gubler, W. D. 2010. Pathogenicity of Diatrypaceae species in grapevines in California. Plant Dis. 94:867-872.

38. Trouillas, F. P., Úrbez-Torres, J. R., and Gubler, W. D. 2010. Diversity of diatrypaceous fungi associated with grapevine canker diseases in California. Mycologia 102:319-336.

39. Trouillas, F. P., Úrbez-Torres, J. R., Peduto, F., and Gubler, W. D. 2010. First report of twig and branch dieback of English walnut (Juglans regia) caused by Neofusicoccum mediterraneum in California. Plant Dis. 94:1267.

40. Udayanga, D., Xingzhong, L., McKenzie, E. H. C., Chukeatirote, E., 
Bahkali, A. H. A., and Hyde, K. D. 2011. The genus Phomopsis: Biology, applications, species concepts and names of common phytopathogens. Fungal Divers. 50:189-225.

41. United States Department of Agriculture, National Agricultural Statistics Service, California Field Office, California Agricultural Statistics, Crop Year 2010, 39-65.

42. United States Department of Agriculture, National Agriculture Statistics Service. 2007 Census of Agriculture California State and County Data.

43. Úrbez-Torres, J. R. 2011. The status of Botryosphaeriaceae species infecting grapevines. Phytopathol. Mediterr. 50:5S-44S.

44. Úrbez-Torres, J. R., Battany, M., Bettiga, L. J., Gispert, C., McGourty, G., Roncoroni, J., Smith, R. J., Verdegaal, P., and Gubler, W. D. 2010. Botryosphaeriaceae spore trapping studies in California vineyards. Plant Dis. 94:717-724.

45. Úrbez-Torres, J. R., and Gubler, W. D. 2009. Pathogenicity of Botryosphaeriaceae species isolated from grapevine cankers in California. Plant Dis. 93:584-592.

46. Úrbez-Torres, J. R., Leavitt, G. M., Voegel, T. M., and Gubler, W. D. 2006. Identification anddistribution of Botryosphaeria spp. associated with grape- vine cankers in California. Plant Dis. 90:1490-1503.

47. Úrbez-Torres, J. R., Peduto, F., and Gubler, W. D. 2010. First report of grapevine cankers caused by Lasiodiplodia crassipora and Neofusicoccum mediterraneum in California. Plant Dis. 94:785.

48. Úrbez-Torres, J. R., Peduto, F., Striegler, K., Rupe, J. C., Cartwright, R. D., and Gubler, W. D. 2012. Characterization of fungal pathogens associated with grapevine trunk diseases in Arkansas and Missouri. Fungal Divers. 52:169-189.

49. Van Niekerk, J. M., Groenewald, J. Z., Farr, D. F., Fourie, P. H., Halleen, F. and Crous, P. W. 2005. Reassessment of Phomopsis species on grapevines. Australas. Plant Pathol. 34:27-39.

50. Vossen, P. M., and Kicenik-Devarenne, A. 2011. UC Cooperative Extension sensory analyses panels enhances the quality of California olive oil. Calif. Agric. 65:8-13.

51. White, T. J., Bruns, T., Lee, S., and Taylor, J. 1990. Amplification and direct sequencing of fungal ribosomal RNA genes for phylogenetics. Pages 315 322 in: PCR Protocols, A Guide to Methods and Applications. M. A. Innis, D. H. Gelfand, J. J. Sninsky, and T. J. White, eds. Academic Press, San Diego, CA. 